\title{
Three-Dimensional Carbuncles and Euler Fluxes
}

\author{
Keiichi Kitamura $^{*}$ and Eiji Shima ${ }^{\dagger}$ \\ Japan Aerospace Exploration Agency (JAXA), Sagamihara, Kanagawa, 229-8510, Japan \\ and \\ Philip L. Roe \\ University of Michigan, Ann Arbor, MI 48109, USA
}

\begin{abstract}
Hypersonic flow computations have proved to be very troublesome due to appearance of shock anomalies (instabilities and oscillations), such as carbuncle phenomenon. These anomalies are categorized into one-dimensional and multidimensional modes, and they both arise from many factors and their combinations. Accurate prediction of hypersonic heating, a key issue in hypersonic flow computations, is therefore challenging especially for three dimensions. In the preceding studies, we focused on one- or two-dimensional shock anomalies and heating. In the present study, we extend these discussions to three dimensions motivated by the following reasons: 1) Naively, multidimensional shock anomalies are considered to develop more likely in three dimensions than in two dimensions, but it cannot be proved mathematically, nor has it been numerically demonstrated; specifically, it is not clear yet whether the third dimension plays another role which is absent in two dimensions. 2) Most of proposed remedies for multidimensional anomalies had been tested in one- or two-dimensional setups in the literature, but it is not guaranteed whether multidimensional dissipations of such methods actually work well in three dimensions. To clarify those, we conducted numerical experiments proposed by the authors along with other benchmark tests in three dimensions for five popular fluxes. The results show that three-dimensional anomalies are quite complicated particularly in their development, and they can either be partly removed or (even worse) enhanced by multidimensional dissipations. Therefore, although most of the existing Euler fluxes are designed based on one or two dimensions and readily extendable to three-dimensions, it is desirable to invent a new method in consideration of the presented three-dimensional behaviors of the captured shock.
\end{abstract}

\section{Nomenclature}

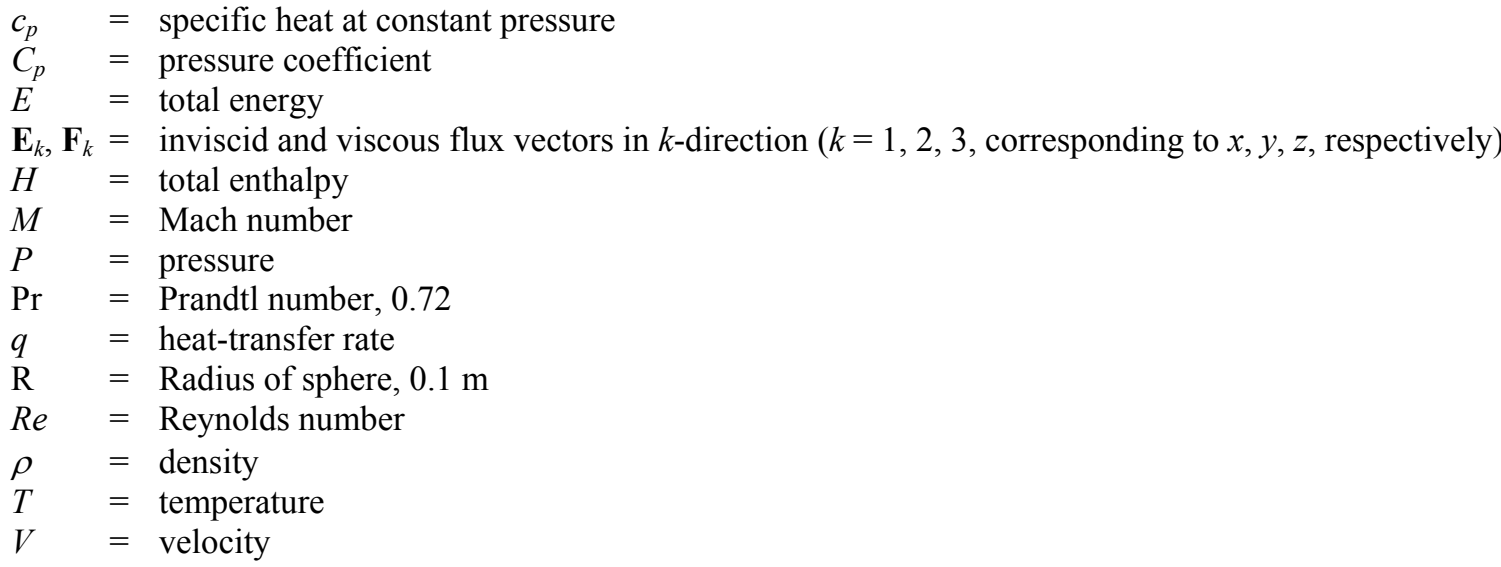

\footnotetext{
* Researcher, JAXA's Engineering Digital Innovation (JEDI) Center, 3-1-1 Yoshinodai, AIAA Member.

${ }^{\dagger}$ Senior Researcher and Director, JAXA’s Engineering Digital Innovation (JEDI) Center, 3-1-1 Yoshinodai, AIAA Member.

* Professor, Department of Aerospace Engineering, AIAA Fellow.
} 


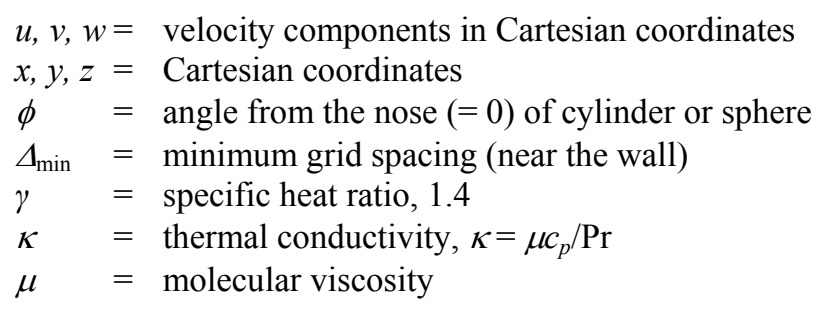

\section{Subscripts}

cell $=$ value based on the minimum grid spacing

$\mathrm{F}-\mathrm{R}=$ Fay-Riddell's theoretically predicted value

$\mathrm{W}=$ value on the wall

$\infty \quad=$ freestream value

$0=$ stagnation value

$1=$ post-shock value

$2=$ pre-shock value

\section{Introduction}

$\mathrm{H}$ YPERSONIC flow computations have proven to be very troublesome due to appearance of shock anomalies (instabilities or oscillations), such as carbuncle phenomenon ${ }^{1}$ (Figs. 1 and 2). Such anomalies arise from the following factors and their combinations ${ }^{2}$ : flow conditions (Mach number, Reynolds number, and the ratio of specific heats), mesh (size, aspect ratio, etc.), and numerical methods (flux function, accuracy, etc.). The authors ${ }^{3}$ recently reported that any flux functions can lead to those anomalous solutions depending on the shock location relative to grid lines. Moreover, they made clear that there are at least two causes of the shock anomalies: one is a onedimensional effect and the other is a multidimensional one. The former appeared to be alleviated by adding dissipation to the shock-normal (1D) direction; whereas the latter could be suppressed by multidimensional dissipation terms that are usually effective to the shock-perpendicular (MultiD) direction. ${ }^{4-10}$ However, when both of the two causes arise at the same time, these dissipations do not work well. Thus, a flux function which is free from those two kinds of instabilities is needed, although we do not have had it yet to the best of the authors' knowledge.

Accurate prediction of hypersonic heating, a key issue in hypersonic flow computations, is therefore challenging, ${ }^{11,12}$ especially for three-dimensional, complex geometries. For heating computations, the authors ${ }^{13}$ suggested the use of flux functions satisfying the following three properties:

I. Shock stability/robustness (i.e., free from both 1D and Multi-D anomalies)

II. Conservation of total enthalpy (and hence, total temperature)

III. Resolving boundary-layer (and hence, temperature gradient)

Unfortunately, it turned out that we had no flux perfectly satisfying all the properties. Nevertheless, the criteria introduced therein for hypersonic heating computations and the classification of Euler fluxes are considered useful pieces of information in choosing/developing Euler fluxes (for details, please see [13]).

As reviewed above, in the preceding papers, ${ }^{3,13}$ we focused on one-dimensional or two-dimensional (2D) issues. In the present study, we will extend these discussions to three dimensions (3D) motivated by the following reasons:

1. Naively, Multi-D shock anomalies are considered to develop more likely in 3D. Specifically, it is not still clear whether the third dimension (added dimension into a 2D setup) plays another role which is absent in 2D. 
2. Most of proposed remedies ${ }^{4-8}$ for shock anomalies had been tested in $1 \mathrm{D}$ and $2 \mathrm{D}$ setups in the literature with the claim that "3D extension is straightforward." It is true in a mathematical sense, however, in conjunction with 1., it is questionable whether Multi-D methods developed according to such a claim actually work well for 3D problems.

3. It is already known to be troublesome to extend some of Multi-D methods developed in 2D considerations to 3D. Yoon et al. ${ }^{10}$ stated in their recent work that the difficulties in 3D extension encountered by their 2Dbased limiter are due to the fact that "cells do not belong to the same plane," for instance. Moreover, from the authors' experience, a Multi-D hybrid flux using two vectors of normal and parallel to the shock ${ }^{6}$ can face a difficulty in uniquely determining the shock-parallel direction in 3D space, though one of those vectors can be (arbitrary) defined: The problem is that the needed dissipation to suppress shock anomalies may differ from one direction to another.

We will conduct numerical experiments proposed by the authors ${ }^{3,13}$ along with benchmark tests ${ }^{12,15}$ for popular or recently-developed Euler fluxes in 3D. The final remarks will be presented with regard to new insights into threedimensional shock anomalies, and to further improvements or developments of flux functions.

\section{Computational Method}

\section{A. Governing Equations}

The governing equations are the compressible Euler or Navier-Stokes equations:

$$
\begin{aligned}
& \frac{\partial \mathbf{Q}}{\partial t}+\frac{\partial \mathbf{E}_{k}}{\partial x_{k}}=0 \quad: \text { Euler } \\
& \frac{\partial \mathbf{Q}}{\partial t}+\frac{\partial \mathbf{E}_{k}}{\partial x_{k}}=\frac{\partial \mathbf{F}_{k}}{\partial x_{k}} \quad: \text { Navier-Stokes }
\end{aligned}
$$

where $\rho$ is density, $u_{i}$ velocity components in Cartesian coordinates, $E$ total energy, $p$ pressure, $H$ total enthalpy $(H=$ $E+(p / \rho))$, and $T$ temperature. The working gas is assumed to be air approximated by the calorically perfect gas model with the specific heat ratio $\gamma=1.4$. The Prandtl number is $\operatorname{Pr}=0.72$. The viscosity $\mu$ is calculated by the Sutherland's formula, and the Stokes' hypothesis is employed: that is, $\lambda=-2 \mu / 3$.

\section{B. Computational Method}

The following methods are used for computations herein, if not mentioned otherwise.

As for spatial discretization, the primitive variables at each cellinterface are simply interpolated from the cell-center values (first-order) for inviscid cases, or second-order accuracy is guaranteed by Van Albada-limited ${ }^{16} \quad$ MUSCL reconstruction ${ }^{17}$ for viscous cases. Then, inviscid fluxes at the cell-

Table 1. Classification of Euler Fluxes Based on Three-Properties for Hypersonic Heating ${ }^{13}$

\begin{tabular}{l|c|c|c|c}
\hline \multicolumn{1}{c|}{ Flux Functions } & Group 1 & Group 2 & Group 3 & Group 4 \\
\cline { 2 - 5 } & $\begin{array}{c}\text { Roe } \\
\text { (E-Fix) }\end{array}$ & Van Leer & Hänel & $\begin{array}{c}\text { AUSM+, } \\
\text { AUSMPW+ }\end{array}$ \\
\hline $\begin{array}{l}\text { I. Shock Stability/ } \\
\text { Robustness }\end{array}$ & Poor & Good & Good & Fair \\
\hline II. H-preserving & $\mathrm{N}$ & $\mathrm{N}$ & $\mathrm{Y}$ & $\mathrm{Y}$ \\
\hline III. B-L Resolution & $\mathrm{Y}$ & $\mathrm{N}$ & $\mathrm{N}$ & $\mathrm{Y}$ \\
\hline
\end{tabular}


interface are calculated from Roe (E-Fix) (Roe ${ }^{18}$ with Harten's entropy-fix ${ }^{19}$ ), Van Leer's FVS, ${ }^{20}$ Hänel, ${ }^{21}$ AUSM $+{ }^{22}$ or AUSMPW $+{ }^{5}$ Roe's FDS (Group 1) is, for example, less dissipative, though it is known to be vulnerable to shock anomalies (e.g., carbuncle phenomenon). ${ }^{2,3}$ Van Leer's FVS (Group 2), on the other hand, had been known to be free from such shock anomalies, but actually exhibit them in extreme cases. ${ }^{13}$ Hänel (Group 3 ) is a variant of Van Leer's FVS, having total enthalpy conserving property. AUSM+ (Group 4), which can be regarded as a mixture of FDS and FVS, is more stable than Roe's flux, ${ }^{2,3}$ though it also suffers from shock anomalies under certain conditions. ${ }^{3,13}$ AUSMPW+ (Group 4) is an improved AUSM+ equipped with a multidimensional dissipation term. These fluxes were categorized as in Table $1 .{ }^{13}$ Other Multi-D fluxes ${ }^{6-9}$ are also of interest, but not covered in the current paper; instead, we will focus on only representative fluxes chosen from each Group in order to clearly compare behaviors of fluxes in different groups.

These fluxes are briefly described in $2 \mathrm{D}$ forms below.

$\underline{\operatorname{Roe}(\text { E-Fix) }}::^{18,19}$

$\mathbf{F}_{1 / 2}=\mathbf{F}_{L}+\mathbf{F}_{R}-\frac{1}{2} \mathbf{R}|\hat{\Lambda}| \mathbf{L} \Delta \mathbf{Q}$

The $\left({ }^{\wedge}\right)$ stands for Roe-averaged values. $\mathbf{R}$ and $\mathbf{L}$ are right and left eigenvectors, respectively, and $\Lambda$

diagonal matrix of characteristic speeds, with entropy-fix $\lambda_{1,4} \rightarrow 0.5\left(\lambda_{1,4}{ }^{2} / \varepsilon+\varepsilon\right)$ if $\left|\lambda_{1,4}\right|<\varepsilon, \quad \varepsilon=0.2$.

Van Leer's FVS: ${ }^{20}$

$\mathbf{F}_{1 / 2}^{ \pm}= \pm \frac{\rho}{4 c}(u \pm c)^{2}\left[1, \frac{(\gamma-1) u \pm 2 c}{\gamma}, \quad v, \frac{[(\gamma-1) u \pm 2 c]^{2}}{2\left(\gamma^{2}-1\right)}+\frac{v^{2}}{2}\right]^{T}$, for $|M|<1$

Hänel: ${ }^{21}$

$\mathbf{F}_{1 / 2}^{ \pm}= \pm \frac{\rho}{4 c}(u \pm c)^{2}\left[1, \frac{(\gamma-1) u \pm 2 c}{\gamma}, \quad v, \quad H\right]^{T}$, for $|M|<1$

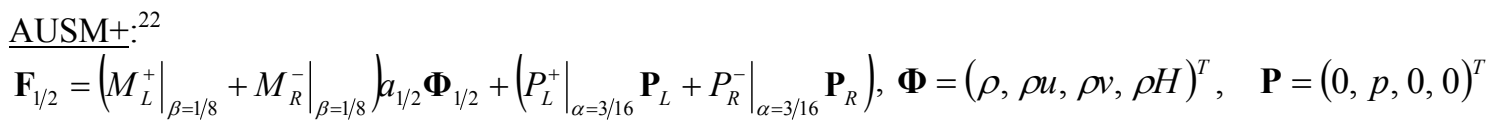

where $a_{1 / 2}=\min \left(\widetilde{a}_{L}, \widetilde{a}_{R}\right), \quad \widetilde{a}=a^{* 2} / \max \left(a^{*},|u|\right)$

$$
\begin{aligned}
& \text { AUSMPW }+:^{5} \\
& \begin{aligned}
& \mathbf{F}_{1 / 2}=\bar{M}_{L}^{+} c_{1 / 2} \mathbf{\Phi}_{L}+\bar{M}_{R}^{-} c_{1 / 2} \mathbf{\Phi}_{R}+\left(\left.P_{L}^{+}\right|_{\alpha=0} \mathbf{P}_{L}+\left.P_{R}^{-}\right|_{\alpha=0} \mathbf{P}_{R}\right), \mathbf{\Phi}=(\rho, \rho u, \rho v, \rho H)^{T}, \quad \mathbf{P}=(0, p, 0,0)^{T} \\
& \text { If } m_{1 / 2}=M_{L}^{+}+M_{R}^{-} \geq 0, \\
& \qquad \bar{M}_{L}^{+}=M_{L}^{+}+M_{R}^{-} \cdot\left[(1-w) \cdot\left(1+f_{R}\right)-f_{L}\right], \\
& M_{R}^{-}=M_{R}^{-} \cdot w \cdot\left(1+f_{R}\right)
\end{aligned}
\end{aligned}
$$

otherwise,

$$
\begin{aligned}
& \bar{M}_{L}^{+}=M_{L}^{+} \cdot w \cdot\left(1+f_{L}\right), \\
& M_{R}^{-}=M_{R}^{-}+M_{L}^{+} \cdot\left[(1-w) \cdot\left(1+f_{L}\right)-f_{R}\right]
\end{aligned}
$$

where

$$
\begin{aligned}
& w=1-\min \left(\frac{p_{L}}{p_{R}}, \frac{p_{R}}{p_{L}}\right)^{3}, \\
& f_{L, R}=\left\{\left(\frac{p_{L, R}}{p_{S}}-1\right) \min \left(1, \frac{\min \left(p_{1, L}, p_{1, R}, p_{2, L}, p_{2, R}\right)}{\min \left(p_{L}, p_{R}\right)}\right)^{2}, \quad p_{S} \neq 0,\right. \\
& 0, \quad \text { otherwise }
\end{aligned}
$$

and $p_{S}=P_{L}^{+} p_{L}+P_{R}^{-} p_{R}$ 
Viscous fluxes are computed by using second-order central difference, while for time integration, first-order Euler explicit method (for inviscid cases) or LU-SGS (for viscous cases) is employed. No turbulence model or real gas model has been used.

\section{Preliminaries: Two-Dimensional Shock Anomalies and Heating}

In this section, along with a few new results, some of our precedent work ${ }^{3,13}$ are reviewed.

\section{A. Two-Dimensional Planar Shock ${ }^{3,13}$}

a) Grid

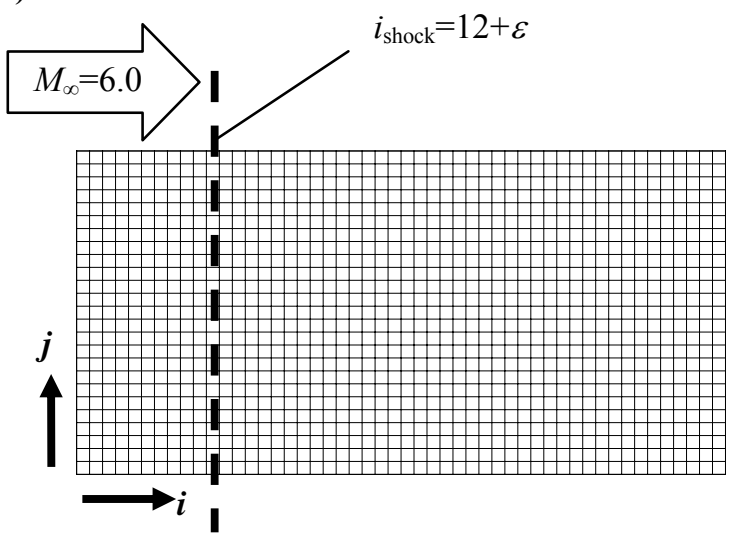

b) Result of Van Leer's FVS

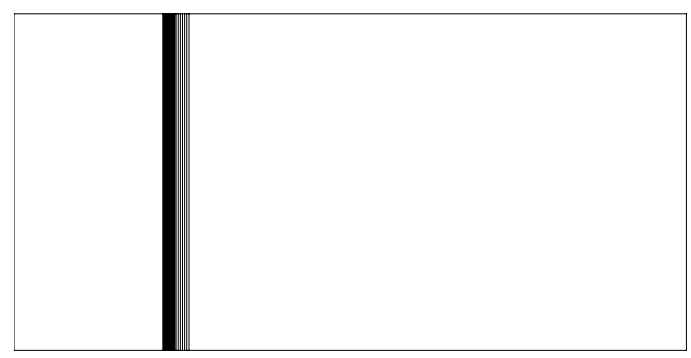

Figure 3. 1-1/2-dimensional steady shock test $(50 \times 25 \text { cells })^{3,13}$.

As shown in Ref. [3], development mechanism of multidimensional (Multi-D) shock anomalies are distinct from their 1D counterpart, although the former anomalies are related to the latter: 1D shock oscillation appears depending on the relative positioning of the shock to the grid line; while the Multi-D oscillations can be triggered by $1 \mathrm{D}$ one in certain conditions; moreover, the Multi-D shock irregularities can develop if the numbers of grid points is increased in the shock perpendicular direction, ${ }^{13}$ as shown in Figs. 3 and 4. This suggested the possibility that the increment of the grid points is equivalent to increment of degrees of freedom for numerical errors to develop.

\section{B. Two-Dimensional}

Shock

\section{Anomaly Test for Cylinder ${ }^{3}$}

The (shock-aligned) grid used in this test had originally been provided by Dr. Jeffery White, NASA Langley.

${ }^{\S}$ We eliminated asymmetry of the received grid first, and made it

a) Grid
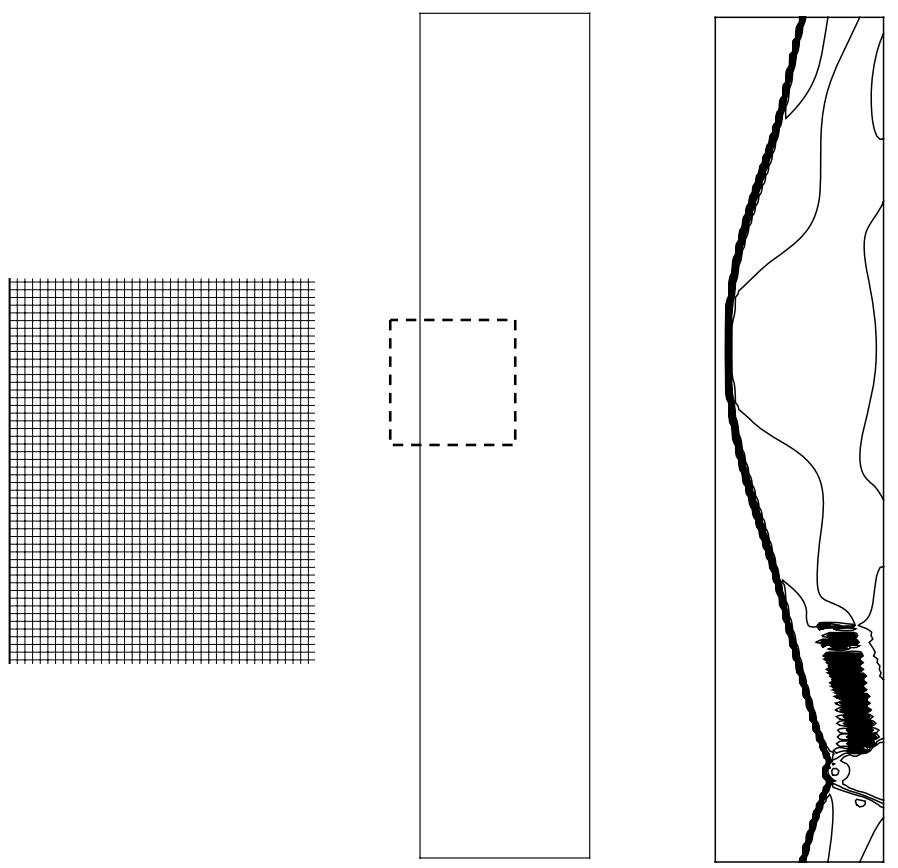

Figure 4. Modified 1-1/2-dimensional test $(50 \times 250$ cells $) .{ }^{13}$

\footnotetext{
$\S$ Private communication with Jeffery White et al., NASA Langley Research Center, Apr. 2007.
} 
slightly dilated so that one grid line closest to the theoretical shock took all the possible locations relative to the shock. The grid system and the computational conditions are:

- Grid: 120 (circumferential) $\times 48$ (wall-normal)

- $\mathrm{CFL}=0.5$

- Computational timesteps: 50,000 steps

- Flow condition: $M_{\infty}=6.0$

In the results, 1D, or (1D-triggered) Multi-D shock anomaly appeared on some grids, whereas it did not emerge on the other grids (Fig. 5). From this difference it had been revealed that the relative positioning of the grid line to the shock played an important role.
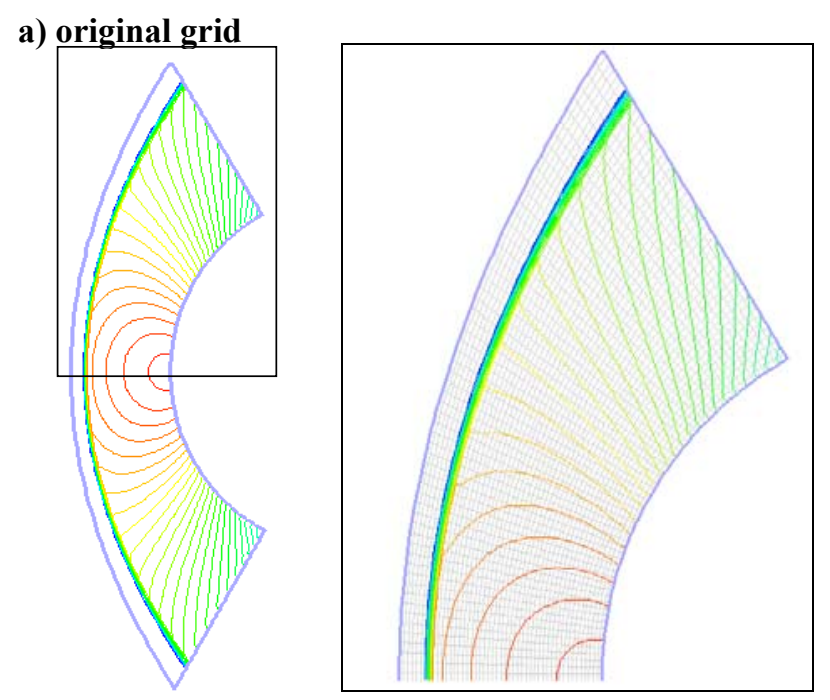

b) modified grid
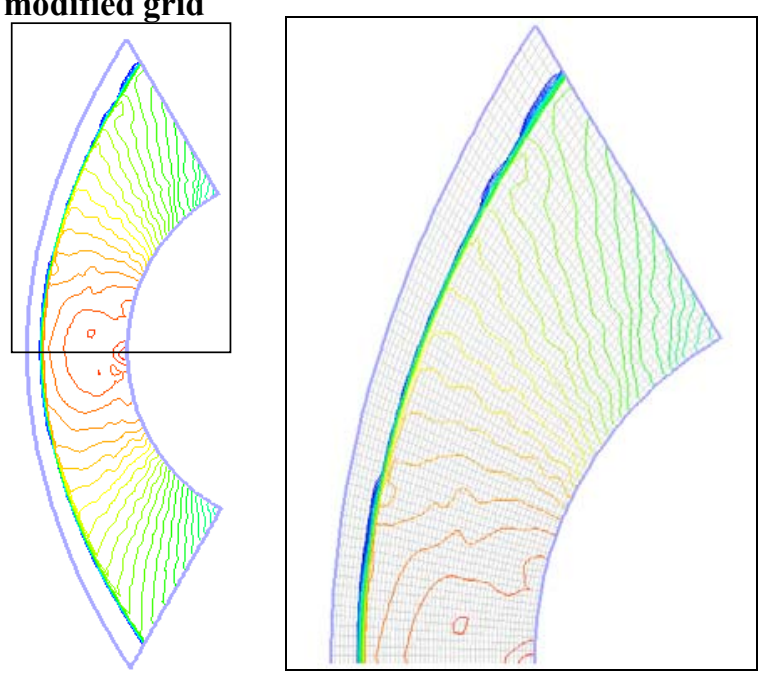

Figure 5. $\quad C_{p}$ contours with grid around $2 \mathrm{D}$ cylinder (Roe, second-order). ${ }^{3}$

\section{LAURA Benchmark ${ }^{9,15}$ : Two-Dimensional Hypersonic Heating Test}

This is a viscous, hypersonic $\left(M_{\infty}=17\right)$ benchmark test used for LAURA and FUN3D $\operatorname{codes}^{15}$, employing a shock-aligned grid. The grid was provided by Dr. Peter Gnoffo, NASA Langley ${ }^{* *}$ as a 3D mesh (appears later), but one slice of it was taken and used here as a 2D version. Then, as in the previous test, we made the same modification on this grid, i.e., the "original grid" was dilated a half-cell width as the "modified grid."

- Grid: $30 \times 64, \Delta_{\min }=2.66 \mathrm{e}-6 \mathrm{~m}$

$-\mathrm{CFL}=200$

- Computational timesteps: 100,000 steps

- Flow conditions: $V_{\infty}=5000 \mathrm{~m} / \mathrm{s}\left(M_{\infty}=17\right), R e=376,930 / \mathrm{m}, \rho_{\infty}=0.001 \mathrm{~kg} / \mathrm{m}^{3}, T_{\infty}=200 \mathrm{~K}, T_{\mathrm{w}}=500 \mathrm{~K}$

With the above setup, the cell Reynolds number is $R e_{\text {cell }}=1.00$, ratio of Pitot pressure to free stream pressure $P_{10} / P_{2}=387.6$, and Fay-Riddell' ${ }^{23}$ stagnation heating $46.5 \mathrm{~W} / \mathrm{cm}^{2}$ (slightly smaller than LAURA-predicted value ${ }^{9}$ of $52 \mathrm{~W} / \mathrm{cm}^{2}$ ).

Computed results are summarized in Figs. 6-9. One can see that Roe (E-Fix) suffered from shock anomaly in the modified grid (Fig. 7a) (although the calculation almost converged as shown in Fig. 9c), but not in the original grid (Fig. 6a). This anomaly clearly affected surface pressure and, more severely, surface heating rates (more than $20 \%$ overestimation) (Figs. 8a, 8b, 9a, and 9b). Other flux functions yielded symmetry and stable (almost converged) solutions with exception of AUSM+ (oscillatory results on either of the two grids). Van Leer and Hänel resulted in poor predictions of heating, as expected. In addition, we point out here that a non-shock aligned grid produces similar results as shown in [13].

Having presented our recent results in two dimensions, we will proceed to three-dimensional discussions.

\footnotetext{
** Private communication with Peter Gnoffo, NASA Langley Research Center, Mar. 2009.
} 


\section{Three-Dimensional Hypersonic Heating Tests}

\section{A. Three-dimensional Planar Shock}

This is an extended planar shock test (from III-A) to three dimensions.

- Test \#1: The grid consists of 10 of the 2D grids (Fig. 3a) stacked in the additional dimension $(50 \times 25 \times 10$ cells, Fig. 10a).

- Test \#2: The grid is equivalent to the $2 \mathrm{D}$ grid in Fig. $4 \mathrm{a}$, but used with three-dimensional code $(50 \times 250 \times 1$ cells).

- Test \#3: The grid consists of 10 of the $2 \mathrm{D}$ grids (Fig. $4 \mathrm{a})$ stacked in the additional dimension $(50 \times 250 \times 10$ cells).

All the cells are isotropic, and the computational conditions are the same as the $2 \mathrm{D}$ case, such as $M_{\infty}=6.0$. The initial shock position parameter ${ }^{3}, \varepsilon$, is taken as 0.0 or 0.5 (the initial shock is imposed exactly on a cell-interface

a) Roe (E-Fix)

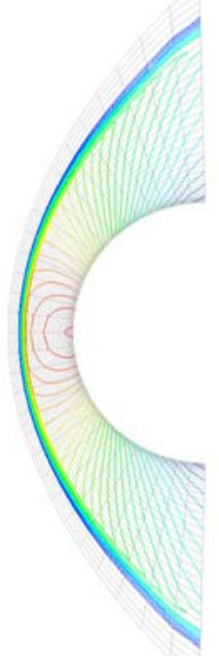

b) Van Leer

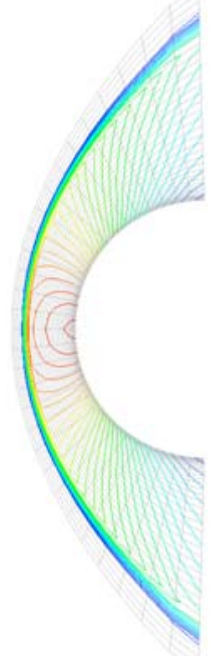

c) Hänel

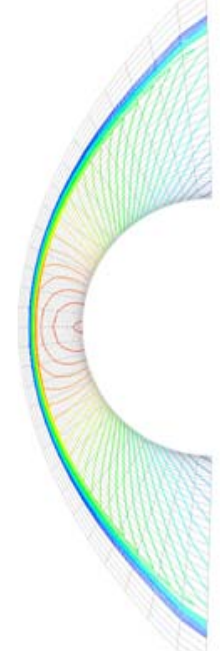

d) AUSM+

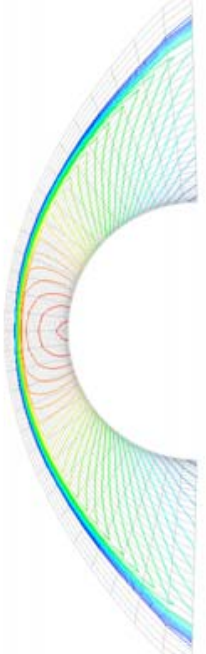

e) AUSMPW+

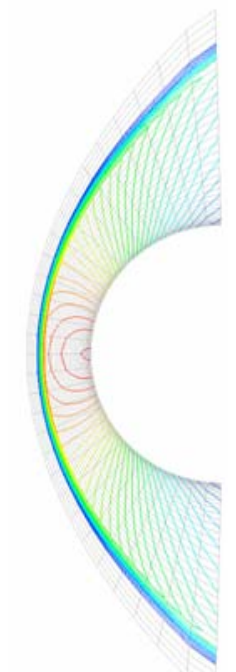

Figure 6. Pressure contours $\left(0<P / P_{\infty}<386\right)$ for circular-cylinder (second-order in space; freestream Mach number $\left.M_{\infty}=17\right)$. [2D, original grid; $30 \times 64$ cells]

a) Roe (E-Fix)

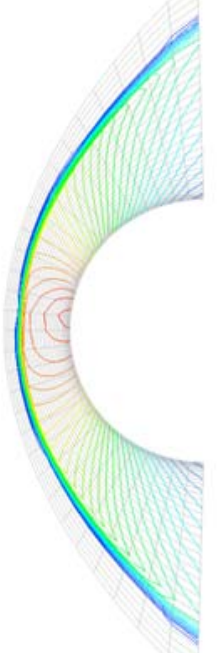

b) Van Leer

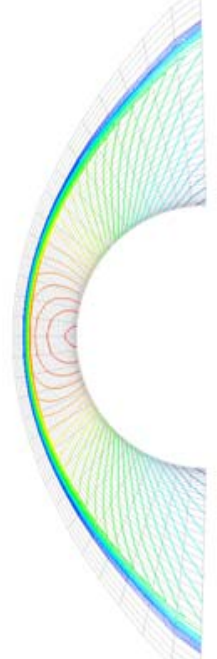

c) Hänel

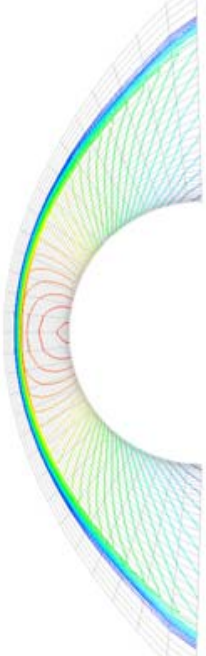

d) AUSM+

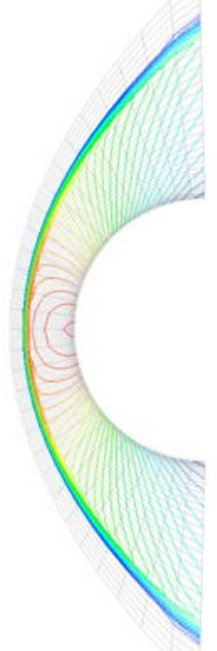

e) AUSMPW+

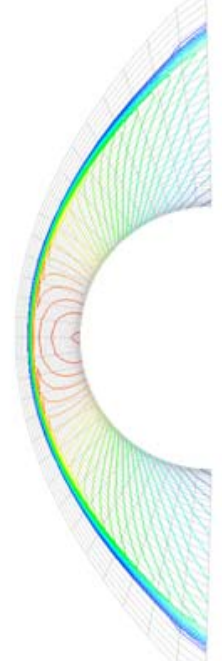

Figure 7. Pressure contours $\left(0<P / P_{\infty}<386\right)$ for circular-cylinder (second-order in space; freestream Mach number $\left.M_{\infty}=17\right)$. [2D, modified (half-cell dilated) grid; $30 \times 64$ cells]. 
when $\varepsilon=0.0$, and at the cell-center when $\varepsilon=0.5$; see Fig. 3a). Then, only selected results will be presented below.

The results for Test $\# 1$ are shown in Figs. 10-13. The Roe (E-Fix) (Fig. 10b) showed total breakdown (carbuncle) in three dimensions. The shock shape is irregular, i.e., the instability occurs in every direction with respect to the shock. Results of Van Leer (Fig. 11), on the other hand, are stable as in the 2D case (Fig. 3b). AUSM+ (Fig. 12) showed a stable result for $\varepsilon=0.0$, but oscillatory for $\varepsilon=0.5$, in consistent with the $2 \mathrm{D}$ cases in Ref. [13]. AUSMPW $+(\varepsilon=0.0)$ once showed a regular oscillation in both directions perpendicular to the captured shock (Fig. 13a), but later the original planar shape was recovered (Figs. 13b and 13c). This recovery seems to be due to Multi-D dissipation term in AUSMPW+. The similar behavior was already observed in 2D and in another Multi-D flux, ${ }^{6}$ but its effectiveness in 3D (in the current particular case) has been confirmed.

a) Pressure

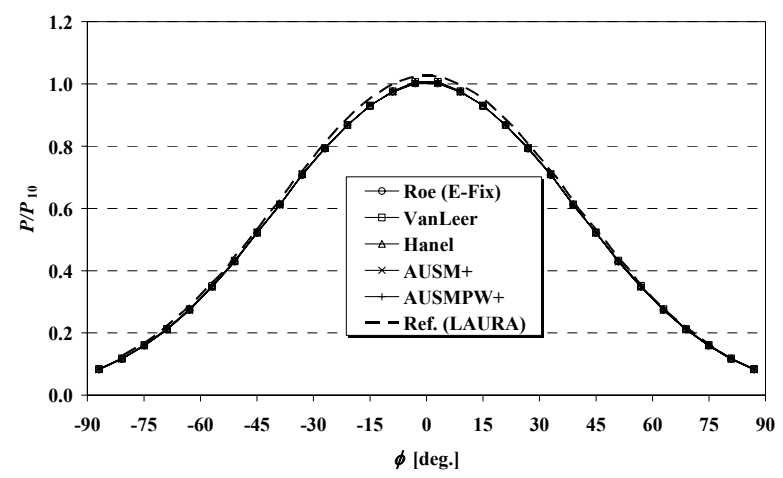

b) Heating

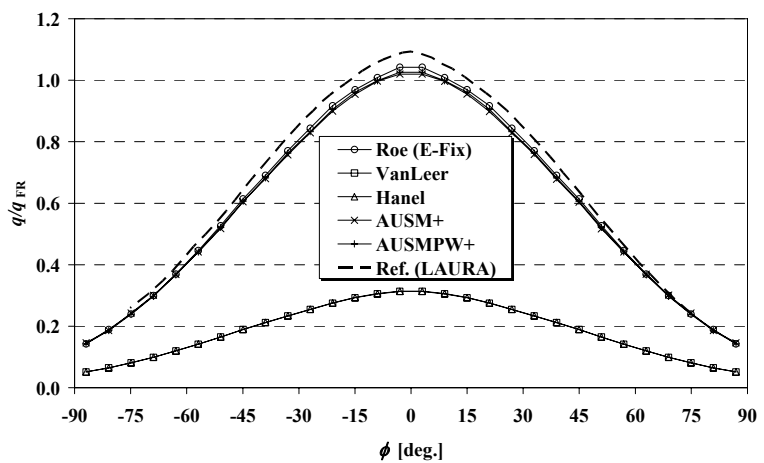

c) L2-norm of density residual

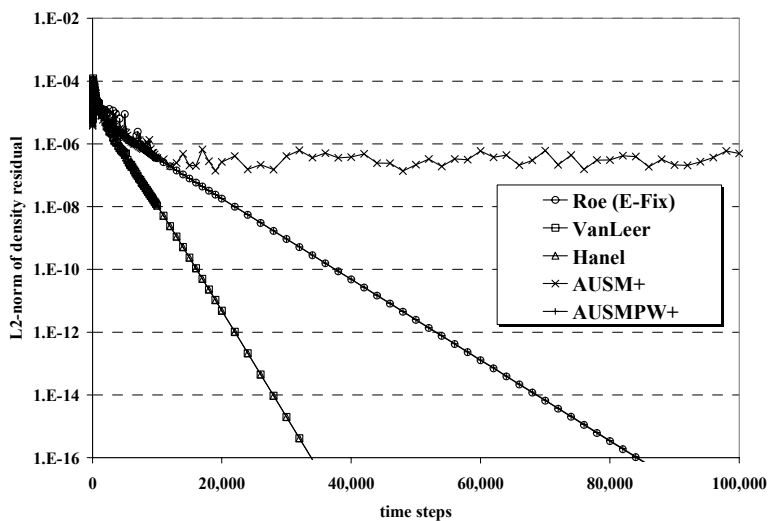

Figure 8. Surface pressure and heating profiles, and residual histories for cylinder (second-order in space; freestream Mach number $\left.M_{\infty}=12\right)$. [2D, original grid]. a) Pressure

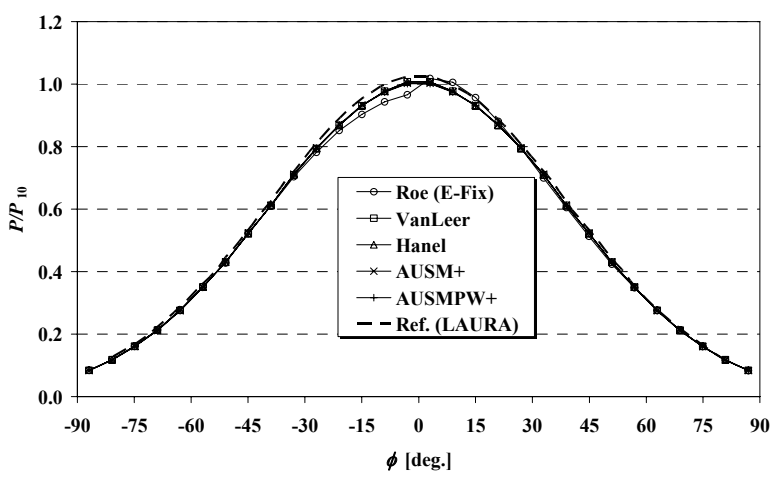

b) Heating

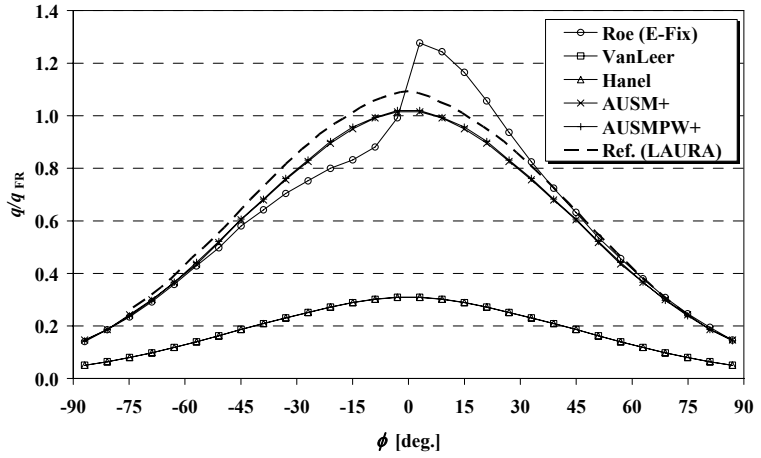

c) L2-norm of density residual

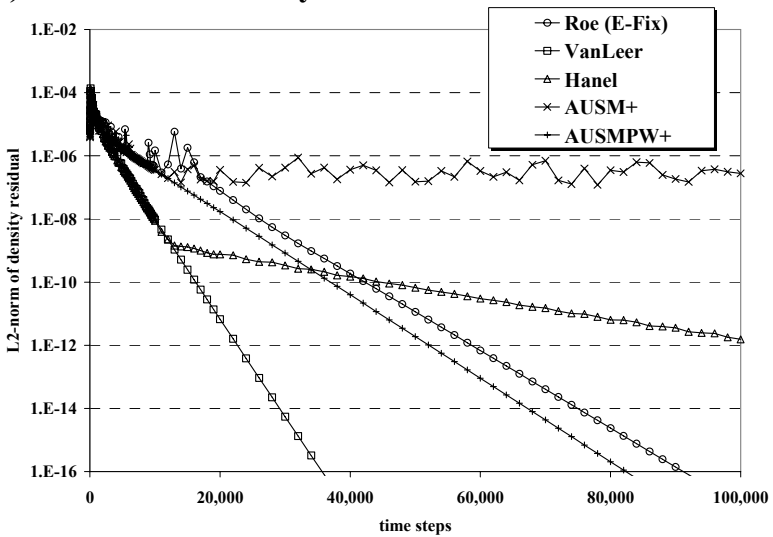

Figure 9. Surface pressure and heating profiles, and residual histories for cylinder (second-order in space; freestream Mach number $M_{\infty}=12$ ). [2D, modified (half-cell dilated) grid]. 
The set of the results for Test $\# 2$ are shown in Figs. 14 (Van Leer) and 15 (AUSMPW+). Not surprisingly, the results of Van Leer are technically the same to those in 2D (Fig. 4), demonstrating that the 3D setup (e.g., ghost cells in the third direction) plays no significant roles. AUSMPW+, known to be less robust than Van Leer, ${ }^{13}$ exhibited earlier and worse development of shock instability than Van Leer. In spite of these unsurprising outcomes, it is interesting to compare the result in Fig. 15 (AUSMPW+ in Test \#2) with those in Fig. 11 (AUSMPW+ in Test \#1). Although the same numbers of cells are used as a total in those two tests, the 'recovery' seen in Test \#1 was not observed in Test \#2, that is to say, the Multi-D term in AUSMPW+ did not work properly in Test \#2. Thus, it is hypothesized that the mechanism of birth and/or growth of (pure) 2D shock anomaly (Test \#2) is significantly different from its 3D counterpart (Test \#1), and so is the needed dissipation to suppress it. If so, even though the dissipation term was effective in both cases to 'detect' the shock, the amount of dissipation was not enough to 'cure' the anomaly in Test \#2. Then, it is worth trying to modify the 'weight function $w$ ' in Eq. (8d), for instance, so that the dissipation is adequately controlled when instability or oscillation arises and gradually grows only in a particular direction. Unfortunately, however, modifications in the following turned out to make no substantial differences in the occurrence of instabilities. This issue seems to need further investigation and left as a future work.

$$
w=1-\min \left(\frac{p_{L}}{p_{R}}, \frac{p_{R}}{p_{L}}\right)^{3} \rightarrow 1-\min \left(\frac{p_{L}}{p_{R}}, \frac{p_{R}}{p_{L}}\right), \quad 1-\min \left(\frac{p_{L}}{p_{R}}, \frac{p_{R}}{p_{L}}\right)^{2}, \quad \text { or } 1-\min \left(\frac{p_{L}}{p_{R}}, \frac{p_{R}}{p_{L}}\right)^{4}
$$

Test \#3 results are presented in Figs. 16 (Van Leer) and 17 (AUSMPW+). Astonishingly, the 3D results in Fig. 16 are more stable than the 2D ones in Fig. 14, but in conjunction with the above hypothesis and the results in Figs. 3 and 4 (III-A), numerical dissipation added by cells in the third direction seemed to have a favorable effect in this case. In AUSMPW+ case (Fig. 17), in contrast, reached an unstable solution: small wiggles appeared at 5,000 steps (Figs. 17a and 17b), the breakdown of the shock shape occurred (during 5,000-10,000 steps, Figs. 17a and 17c) earlier than the 2D case (during 10,000-40,000 steps, Figs. 15b and 15c), and finally, developed instability remained at 40,000 steps (Fig. 17d). Note that the final state in Fig. 17d looks similar to the 2D result (Fig. 15c), rather than another 3D result in Test \#1 (Fig. 13c). Therefore, the Multi-D dissipation term in AUSMPW+ does not seem to be effective in cure of this '2D dominant' anomaly.

a) Grid

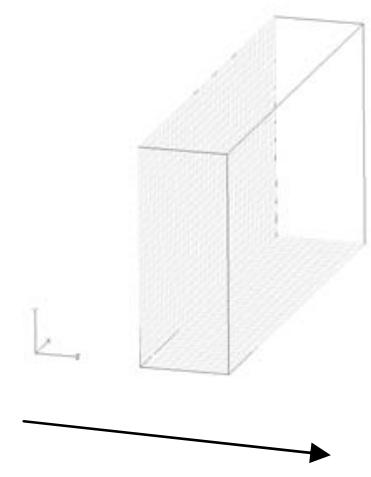

Third Dimension (Direction) b) Roe (E-Fix), $\varepsilon=0.5,5,000$ steps

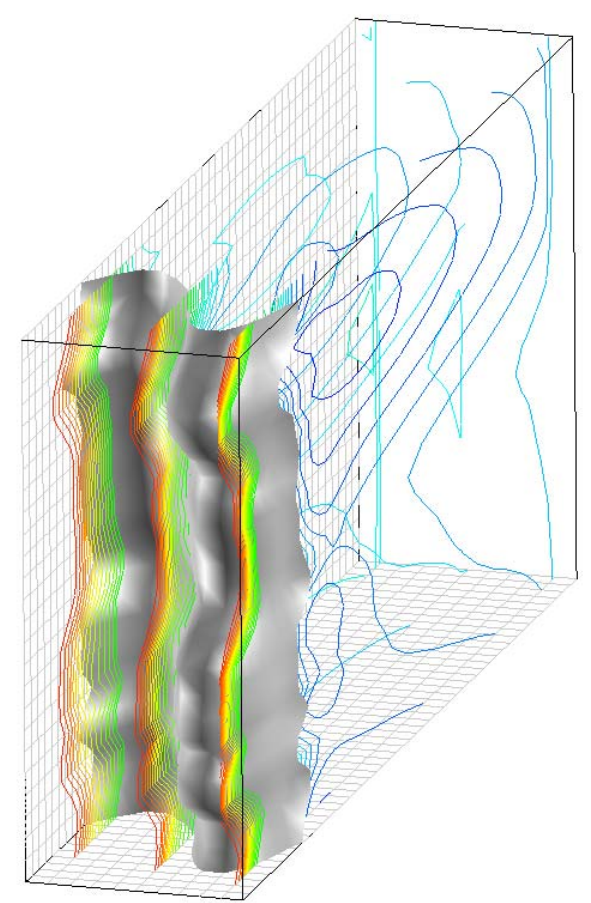

Figure 10. Three-dimensional steady planar shock test \#1 $(50 \times 25 \times 10$ cells) $($ colors: Mach number; gray: $M=1.5$ iso-surface). 
a) $\varepsilon=0.0,5,000$ steps

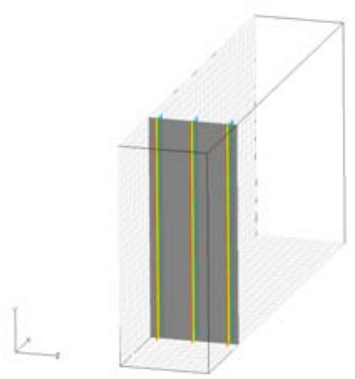

b) $\varepsilon=0.0,40,000$ steps

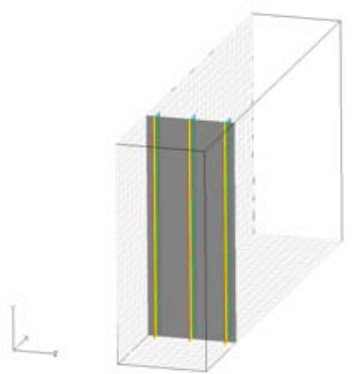

c) $\varepsilon=0.5,40,000 \mathrm{steps}$

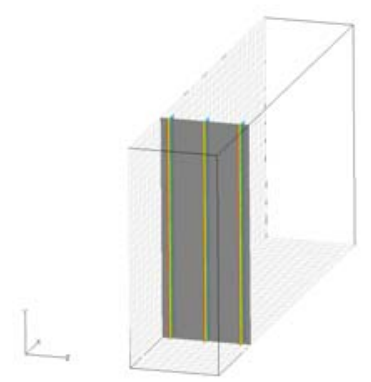

Figure 11. Three-dimensional steady planar shock test \#1 (50 $\times 25 \times 10$ cells), Van Leer.

a) $\varepsilon=0.0,40,000$ steps

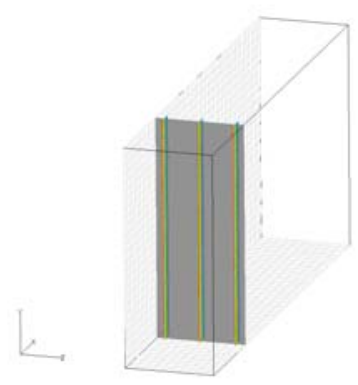

b) $\varepsilon=0.5,40,000$ steps

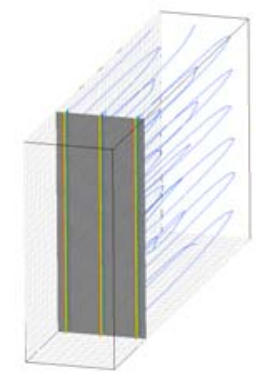

Figure 12. Three-dimensional steady planar shock test \#1 (50 $\times 25 \times 10$ cells $),$ AUSM+.

a) $\varepsilon=0.0,5,000$ steps

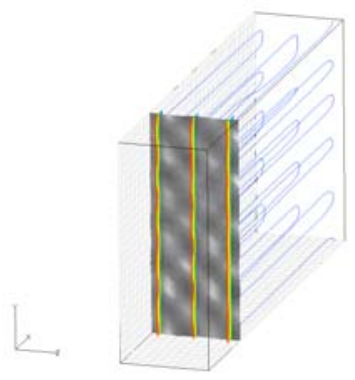

b) $\varepsilon=0.0,10,000$ steps

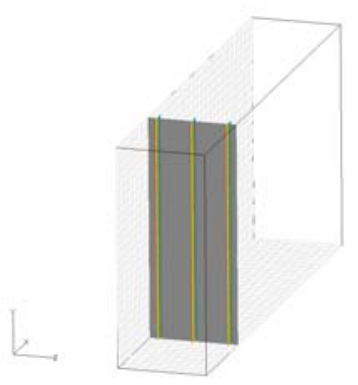

c) $\varepsilon=0.0,50,000$ steps

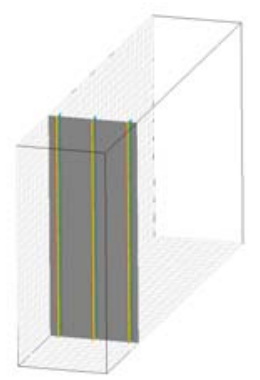

Figure 13. Three-dimensional steady planar shock test \#1 $(50 \times 25 \times 10$ cells $)$, AUSMPW+. 
a) $\varepsilon=0.0,5,000$ steps

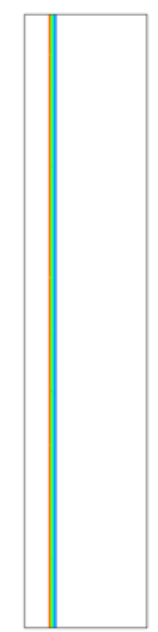

b) $\varepsilon=0.0,40,000$ steps

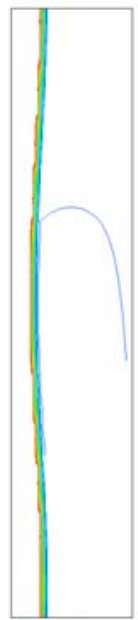

Figure 14. Three-dimensional steady planar shock test $\# 2(50 \times 250 \times 1$ cells $)$, Van Leer.

a) $\varepsilon=0.0,5,000$ steps

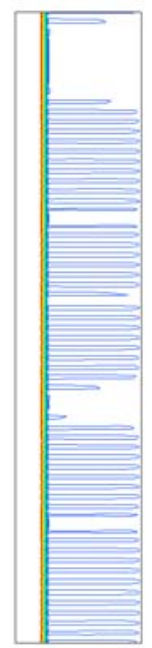

b) $\varepsilon=0.0,10,000$ steps

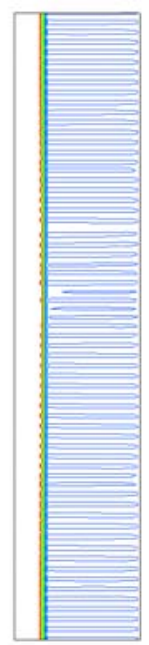

c) $\varepsilon=0.0,40,000$ steps

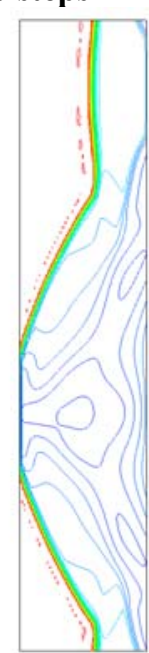

Figure 15. Three-dimensional steady planar shock test \#2 $(50 \times 250 \times 1$ cells $)$, AUSMPW + .

\section{B. Three-dimensional Shock Anomaly Test for Cylinder}

The grid in Section III-B has been extended to three dimensions (100 cells in the third direction).

- Grid: $120 \times 48 \times 100$ (evenly spaced)

- $\mathrm{CFL}=0.5$

- Computational timesteps: 50,000 steps

- Flow condition: $M_{\infty}=6.0$

The results are shown in Figs. 18-20. Figure 18 focused on the development of the "3D carbuncle" for Roe (EFix) case. It is seen from the results that Multi-D shock anomalies developed in every direction: More precisely, the "carbuncle instability" seemed to have occurred in the 2D slice (500 steps, Fig. 18b), while in the third direction, the shock "oscillation" developed (1,500 steps, Fig. 18c). Then, both of these anomalies developed until 5,000 steps (Figs. 18d-f), and this catastrophic solution remained unchanged to 50,000 steps (Fig. 18g), showing the density residual decreased by more than seven orders of magnitude (Fig. 19a). Solutions of this kind, i.e., "converged carbuncles," are also reported in 2D (Fig. 18a or Refs. [3, 6]). 


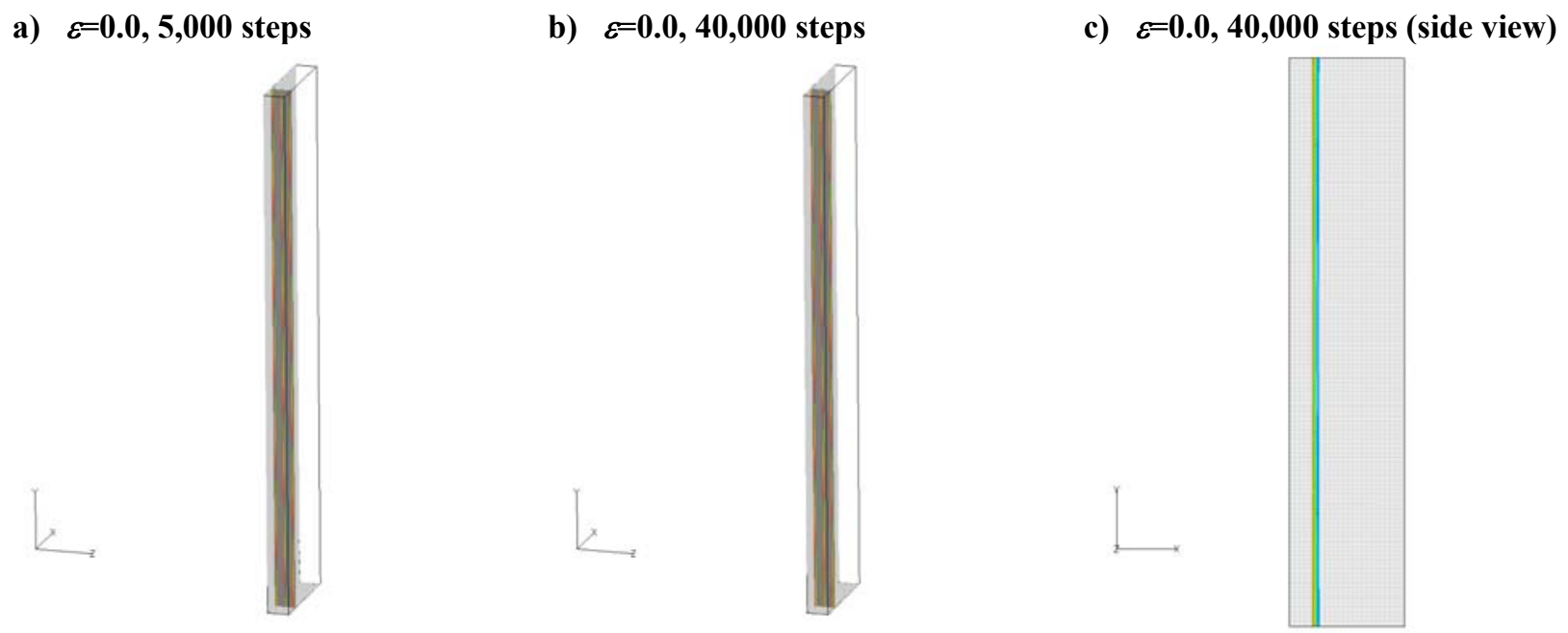

Figure 16. Three-dimensional steady planar shock test $\# 3(50 \times 250 \times 10$ cells $)$, Van Leer.

a) AUSMPW $+, \varepsilon=0.0,5,000$ steps

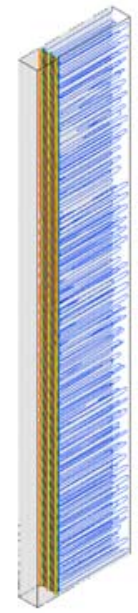

c) $\mathrm{AUSMPW}+, \varepsilon=0.0,10,000$ steps

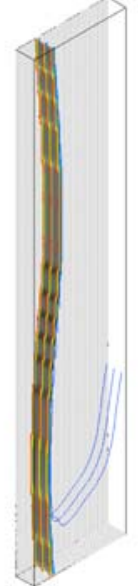

b) AUSMPW $+, \varepsilon=0.0,5,000$ steps (blow-up view)

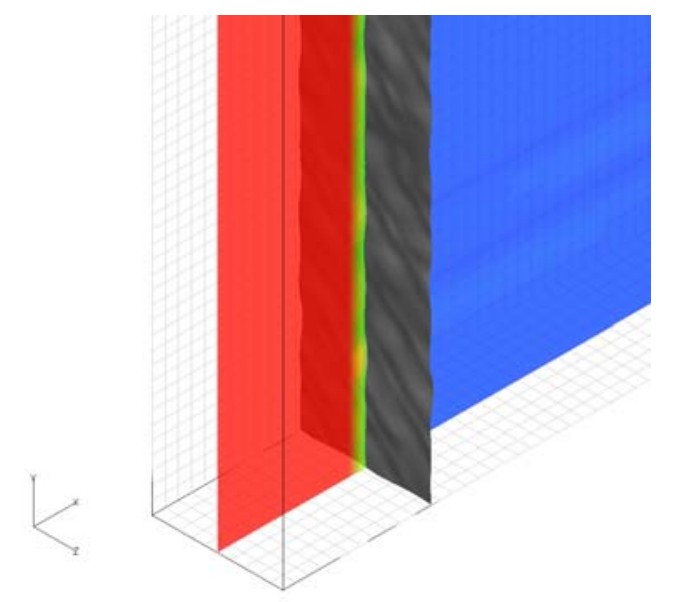

d) AUSMPW+, $\varepsilon=0.0,40,000$ steps

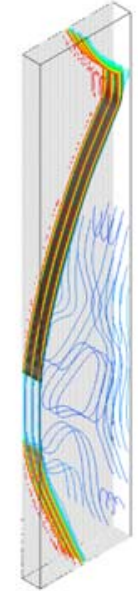

Figure 17. Three-dimensional steady planar shock test $(50 \times 250 \times 10$ cells $)$. 
a) Grid and Reference 2D "Carbuncle" Result ${ }^{3}$
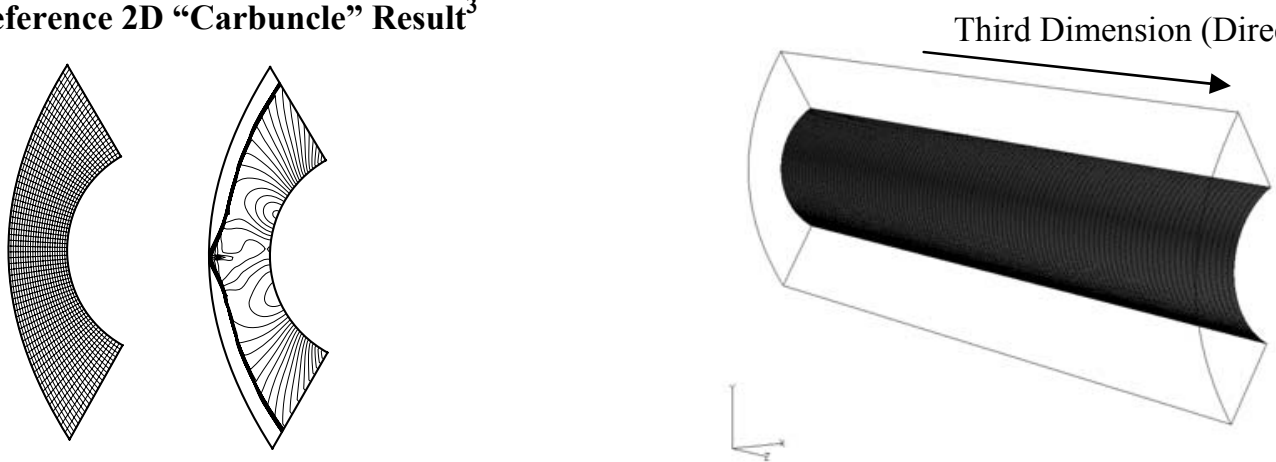

b) 500 steps
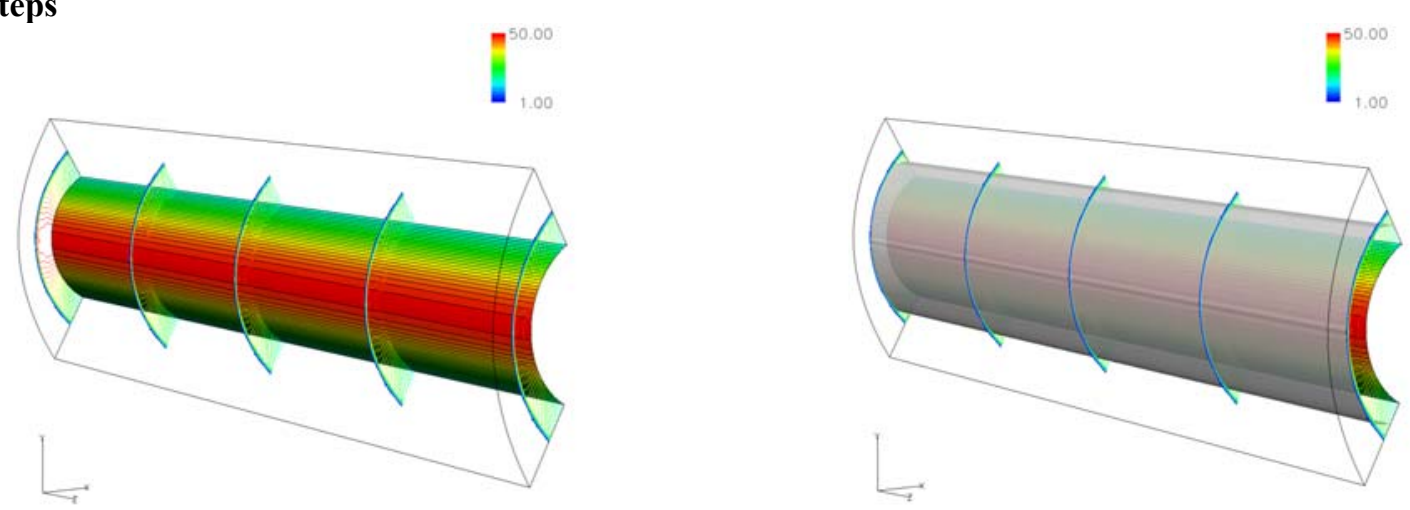

c) 1,500 steps
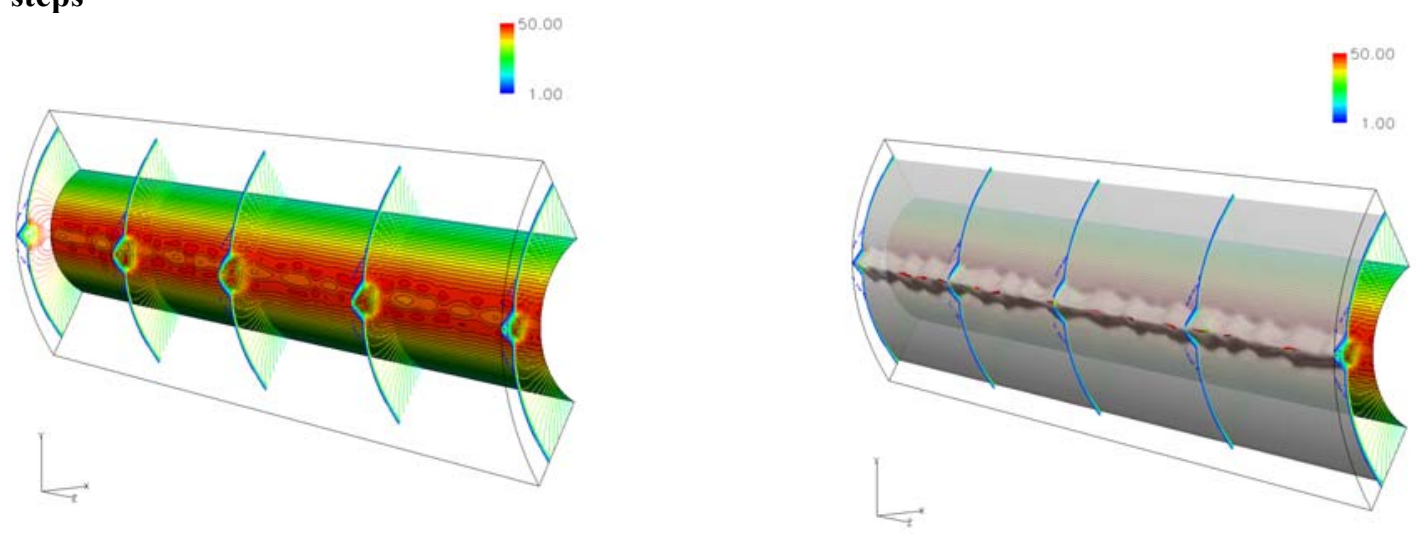

d) 2,000 steps
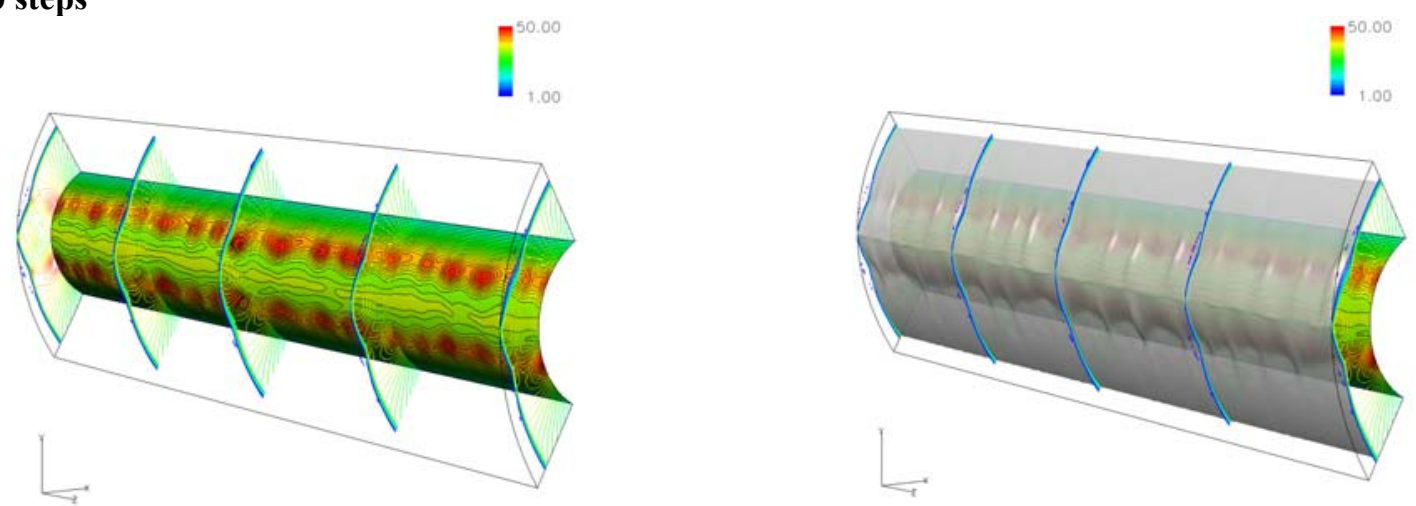

Figure 18. Snapshots of developing 3D carbuncle for circular-cylinder (Roe E-Fix, first-order both in space and time; freestream Mach number $M_{\infty}=6.0$ ); $C_{p}$ contours (left; $0<C_{p}<50$ ) and iso-Mach-number surface (right; $M=1.5$ ) [except for a) of grids and reference 2D result], continued. 
e) 2,500 steps
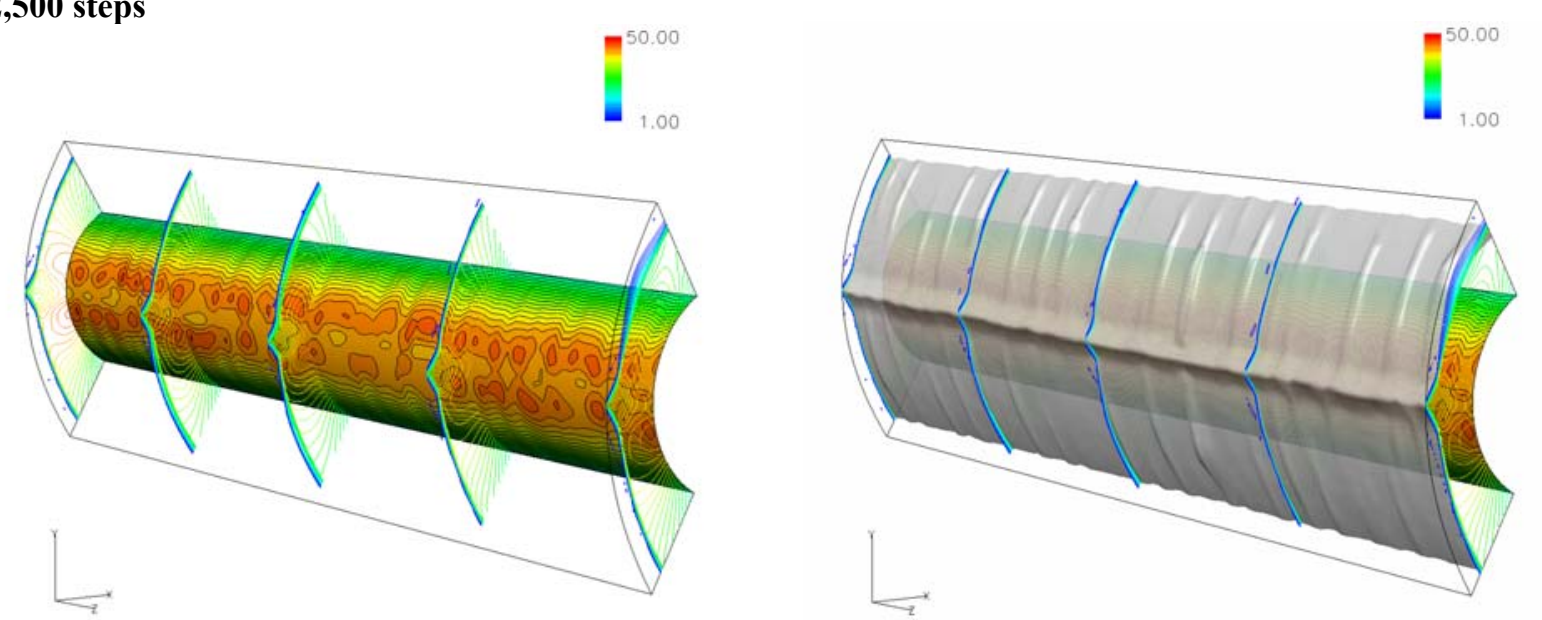

f) 5,000 steps
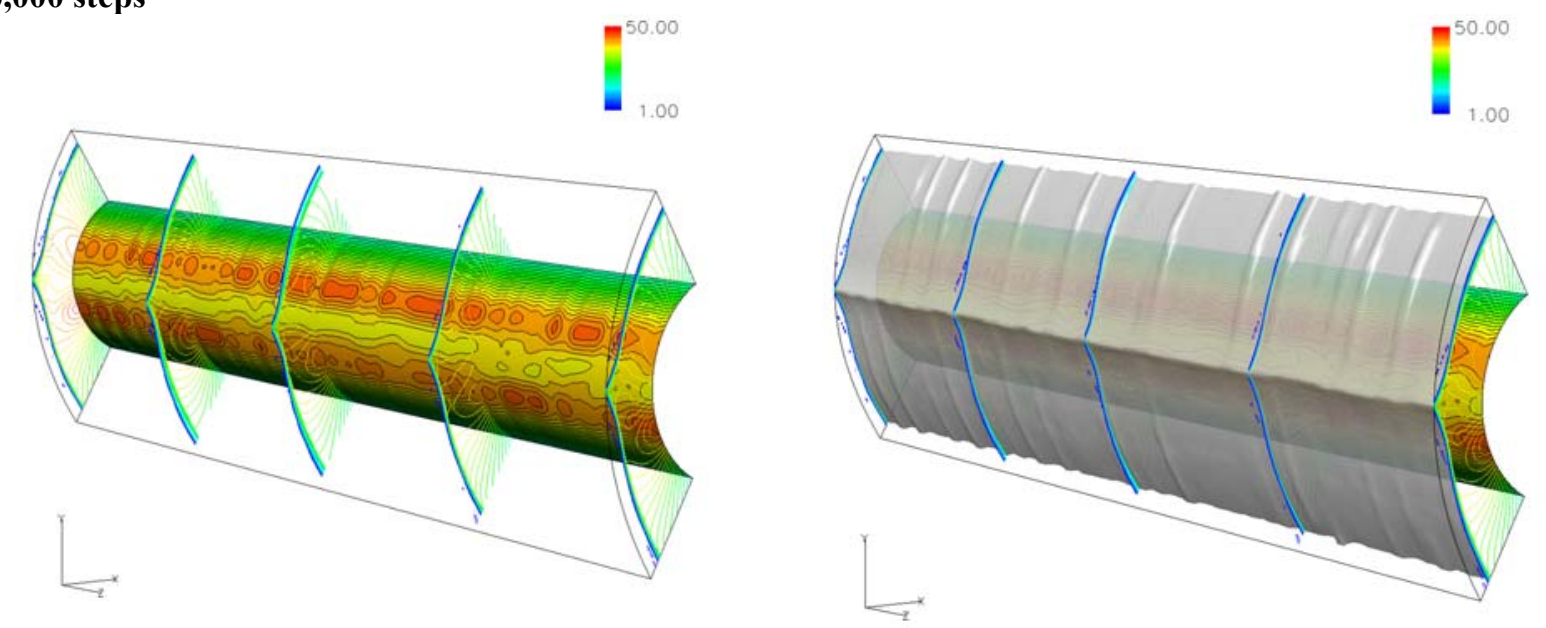

g) 50,000 steps
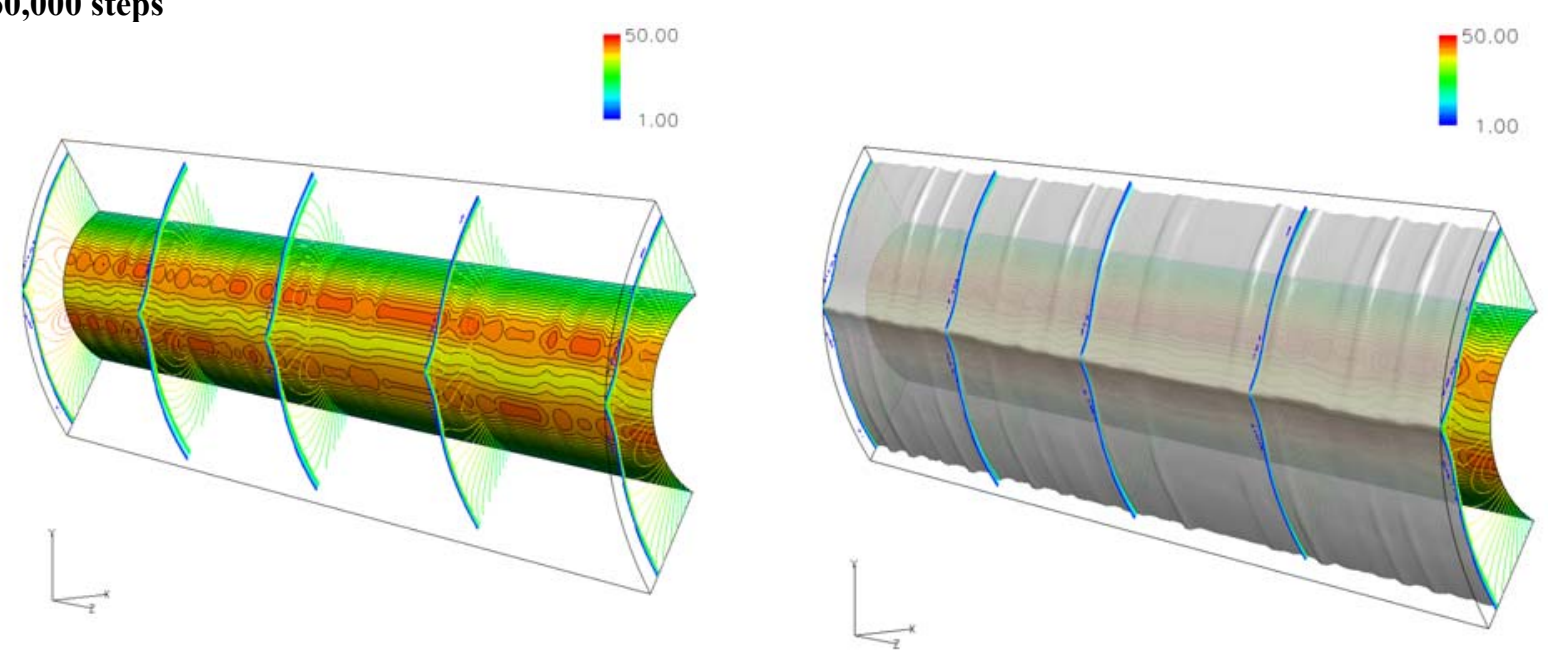

Figure 18. Snapshots of developing 3D carbuncle for circular-cylinder (Roe E-Fix, first-order both in space and time; freestream Mach number $M_{\infty}=6.0$ ); $C_{p}$ contours (left; $0<C_{p}<50$ ) and iso-Mach-number surface (right; $M=1.5$ ) [except for a) of grids and reference 2D result], concluded. 


\section{a) Density residual}

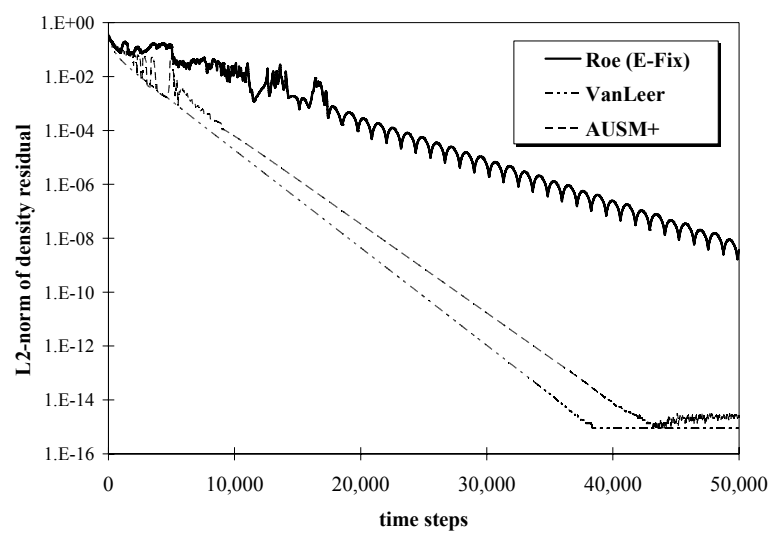

b) Velocity asymmetry in $v$ and $w$ components

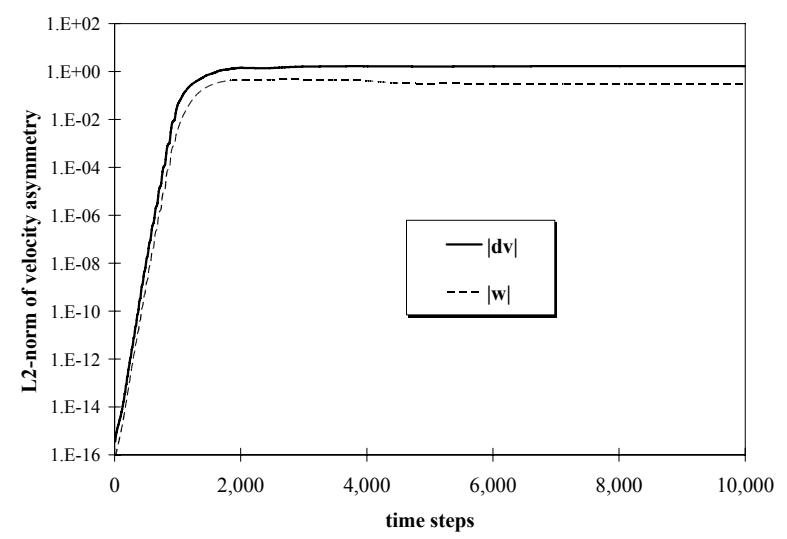

Figure 19. Residual and asymmetry histories for circular-cylinder $\left(M_{\infty}=6.0\right)$. [3D grid]

\section{a) Grid and Reference 2D "Stable" Result (AUSM+) ${ }^{3}$}
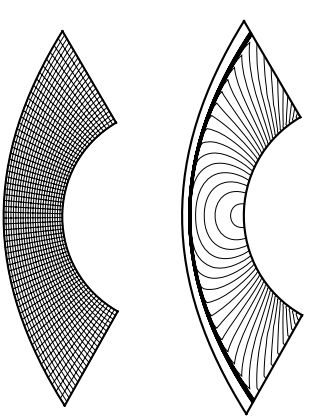

b) AUSM+, 50,000 steps
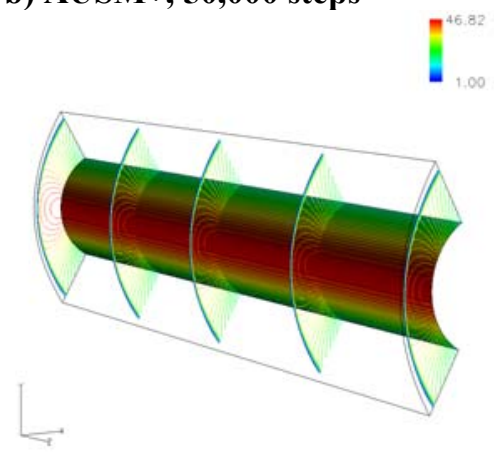

c) Van Leer, 50,000 steps

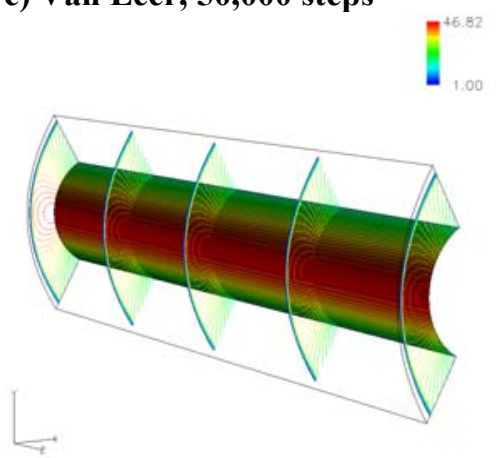

Figure 20. $C_{p}$ contours $\left(0<C_{p}<47\right)$ for circular-cylinder [AUSM+ and Van Leer's FVS, first-order both in space and time; $\left.M_{\infty}=6.0\right]$.

In contrast to the planar shock case (Fig. 10), the shock anomalies in this case appeared to have developed in different modes in different directions, and also, in different rates. These differences seem to be due to the fact that the cells in the third direction are totally the same from one slice to another, while in a slice the cells in the rest of the two dimensions are different. Then, there appeared to be two possibilities:

i) $2 \mathrm{D}$ carbuncle triggered $3 \mathrm{D}$ oscillation, i.e., the appearance of the $3 \mathrm{D}$ oscillation was totally dependent on the 2D carbuncle.

ii) $2 \mathrm{D}$ carbuncle developed faster than $3 \mathrm{D}$ oscillation, i.e., the 3D oscillation gently and subliminally developed while the $2 \mathrm{D}$ instability emerged.

We examined this by comparing L2 norms of $|\Delta v|\left[=\left|v_{\text {upper }}-\left(-v_{\text {lower }}\right)\right|\right.$, i.e., $\left|v(i, j, k)+v\left(i_{\max }+1-i, j, k\right)\right|$, which is velocity difference between cells sharing the same $x$ and $z$ coordinates but having opposite signs in $y$ ] and $|w|$ (in the third dimension), as shown in Fig. 19b. According to this figure, the instabilities both in $y$ and $z$ directions arose from the very beginning of the computation as the form of round-off errors, and they grew exponentially with time. Thus, it is said that the possibility ii) above was almost right with the slight correction that the only difference in the instabilities in those two directions is not in their growth rates but the initial magnitudes. Therefore, in this case, 2D and 3D shock instabilities are strongly related and share the same cause; they developed in the same rate, yet in different appearances.

In AUSM+ or Van Leer results (Figs. 20b and 20c), however, no evidence of shock anomalies is seen: These flow patterns remained stable and symmetry even in the 3D setup. The same holds for even in further extended grid case of $120 \times 48 \times 500$, though the results are omitted. 


\section{LAURA Benchmark Hypersonic Heating Test (3D) ${ }^{9}$}

This is the 3D extension ( 10 cells in the third direction) of Section III-C, viscous, $M_{\infty}=17$, hypersonic heating test, which was previously used, for example, in Ref. [9]. The grid was provided by Dr. Peter Gnoffo, as stated before. The grid system has $30 \times 64 \times 10$ cells, and the flow and computational conditions are the same as in the 2D test (Section III-C).

The results are shown in Figs. $21-26$. According to these figures, the Roe (E-Fix) case was affected by threedimensional effect: the shock shape (Fig. 21a), surface pressure (about 5\%, Fig. 21b), and heating (more than 50\%, Fig. 21c) exhibited asymmetry in the crossflow direction (= the third dimension), as in the previous test (IV-B). It is noteworthy that, in this case, the Roe (E-Fix) result suffered from shock anomaly only in the third dimension and maintained the 2D stable, symmetric solution (Fig. 6a) in each 2D plane; in contrast to the example seen in Fig. 20d in which anomalies appeared in every direction whereas the corresponding $2 \mathrm{D}$ solution was stable. Thus, this result confirms the above-mentioned fact that simply adding one dimension (one degree of freedom) to computational space could lead to an anomalous distribution of physical values in that direction.

Other fluxes showed almost as the same trends as in 2D cases: Van Leer and Hänel reached converged, symmetry solutions with surface heating underestimated; AUSM+, again, suffered from numerical oscillations; AUSMPW + solutions are indistinguishable from the 2D solution.

\section{D. "Challenge" Problem for Sphere (3D) ${ }^{24}$}

This is a genuinely three-dimensional test, referred to as a "challenge" problem in [24] of viscous, hypersonic $\left(M_{\infty}=12\right)$ heating. A shock-aligned grid provided by Dr. Peter Gnoffo (through Dr. Bil Kleb) ${ }^{\dagger \dagger}$ was used. We conducted this test to demonstrate how difficult it is to obtain satisfactory heating profiles by existing methods, and how Multi-D terms work to improve the solutions.

- Grid: $45 \times 160 \times 125=900,000$ cells and $40 \times 40 \times 125=200,000$ cells; totally $1,100,000$ cells (Fig. 27)

- Radius: $\mathrm{R}=0.1 \mathrm{~m}$

$-\mathrm{CFL}=200$

- Computational timesteps: 10,000 steps

- Flow conditions: $V_{\infty}=4167 \mathrm{~m} / \mathrm{s}\left(M_{\infty}=12\right), R e=0.5 \mathrm{e}+6$ (based on radius $\mathrm{R}$ ), $R e_{\text {cell }}=5$ (based on minimum grid spacing $\left.\Delta_{\min }=1 . \mathrm{e}-6 \mathrm{~m}\right), \rho_{\infty}=0.0216 \mathrm{~kg} / \mathrm{m}^{3}, T_{\infty}=300 \mathrm{~K}, T_{\mathrm{w}}=800 \mathrm{~K}$

With the above setup, ratio of Pitot pressure to free stream pressure is $P_{10} / P_{2}=185.9$, and Fay-Riddell's ${ }^{23}$ stagnation heating $550.1 \mathrm{~W} / \mathrm{cm}^{2}$ (smaller than $627 \mathrm{~W} / \mathrm{cm}^{2}$ in [24] and LAURA's prediction ${ }^{9}$ of $590 \mathrm{~W} / \mathrm{cm}^{2}$ ).

Roe (E-Fix) calculation diverged, thus, only the results of Van Leer, AUSM+, and AUSMPW+ are shown. Surface pressure profiles shown in Figs. $27 \mathrm{~b}$ and 28 are almost symmetry and in good agreement with reference data. AUSM+ yielded a very slight glitch at $\phi \approx 15^{\circ}$ (data at an angle $\phi$ stand for all the circumferential data in this test), but this is suppressed by Multi-D term in AUSMPW+. Calculated heating, however, are totally underestimated or asymmetry as shown in Fig. 29, even with this hexahedral mesh. Gnoffo ${ }^{9}$ recently developed a multidimensional version of Roe flux, and the results shown therein were much better than the original Roe, though asymmetry was also seen in heating. Similarly, AUSMPW+, having Multi-D dissipation term, showed here better performance than its precedent version, $\mathrm{AUSM}+$, with respect to preservation of symmetry. These results confirm the necessity of Multi-D terms in Euler fluxes for realization of a method free from Multi-D shock anomalies.

In addition, the heating contours of our results (Figs. 29b and 29c) showed different shapes from Ref. [9] (or Fig. 29d), possibly due to the use of different implicit time integration methods. Fig. 30 shows density residual histories of the present computations, and AUSM+ and AUSMPW+ exhibited around three orders drop in the residual. From the engineering point of view, these solutions can be regarded as 'converged,' and thus we stopped our calculations (although even with this level of residual decrease, it is speculated that shock anomalies grow later ${ }^{3}$ ).

\footnotetext{
${ }^{\dagger \dagger}$ Private communication with Peter Gnoffo and Bil Kleb, NASA Langley Research Center, Mar. 2009.
} 
a) Roe (E-Fix)
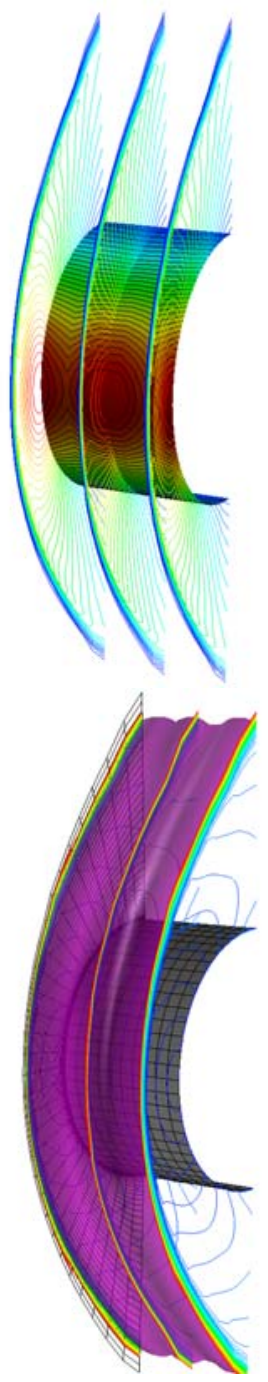

c) Hänel

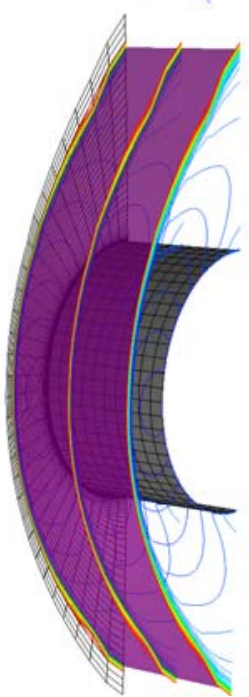

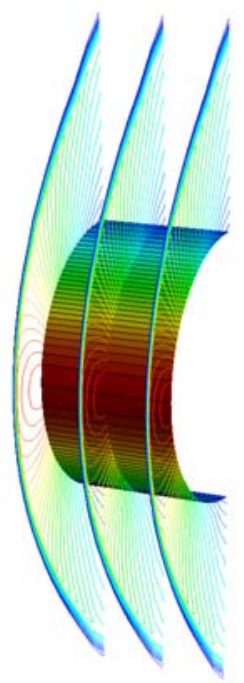

d) AUSM+
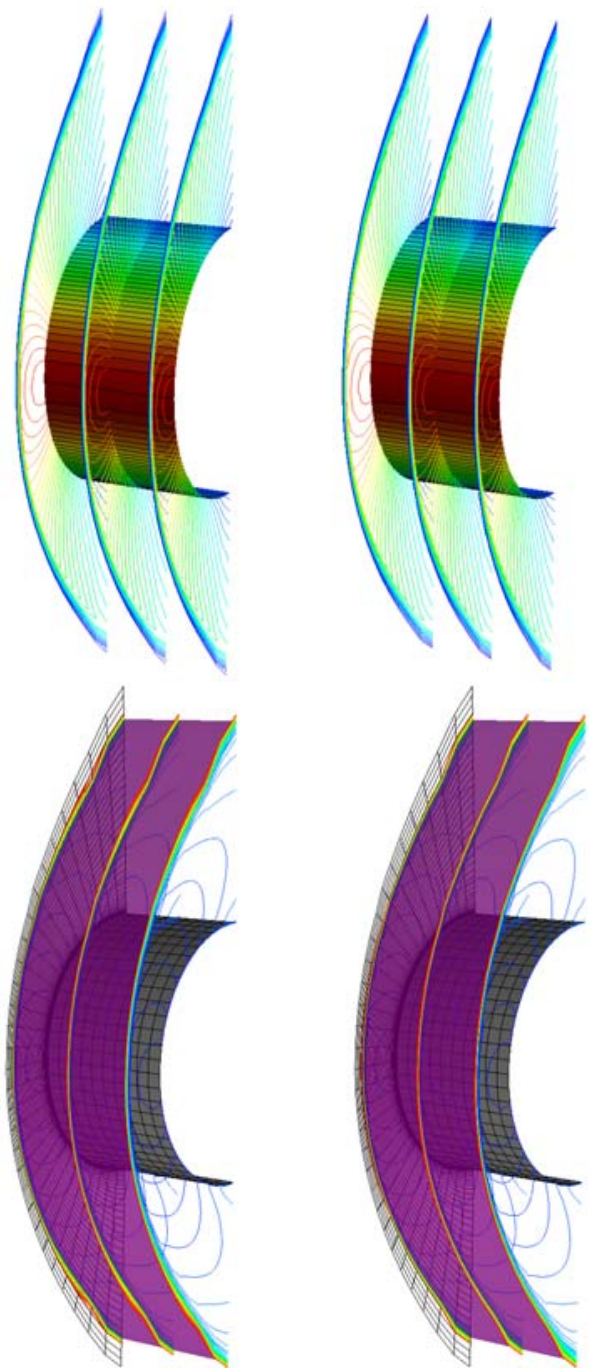

e) AUSMPW+
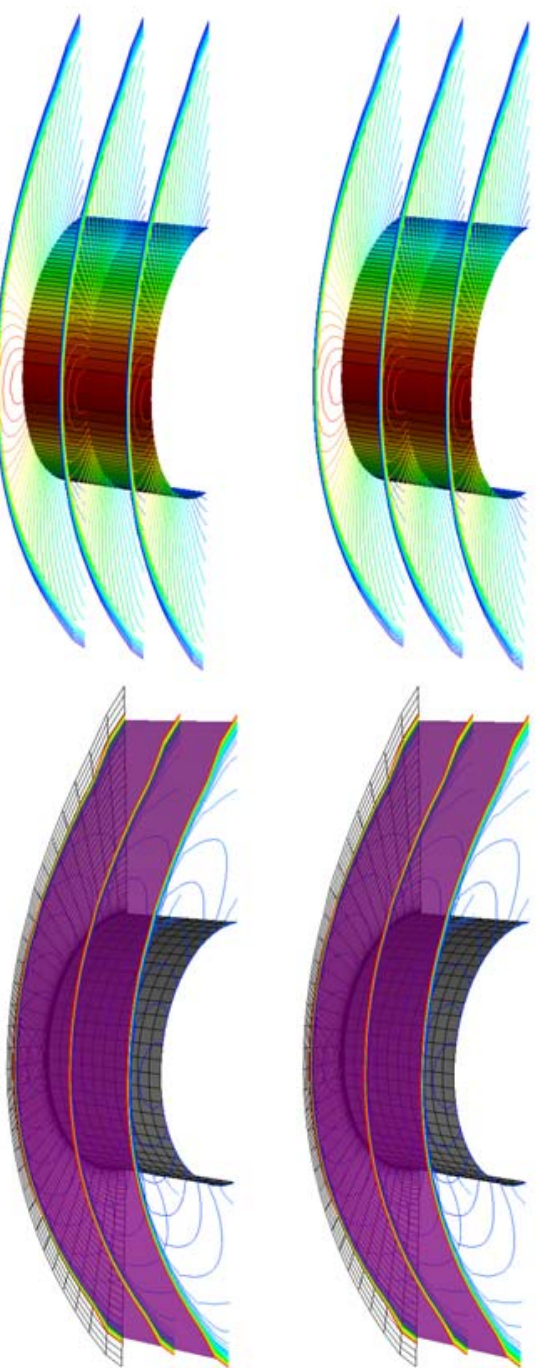

Figure 21. Pressure (top; $0<P / P_{\infty}<390$ ) and Mach number (bottom; $0<M_{\infty}<17$ ) contours for circularcylinder (second-order in space; freestream Mach number $M_{\infty}=17$ ). [3D grid] 


\section{a) Pressure}

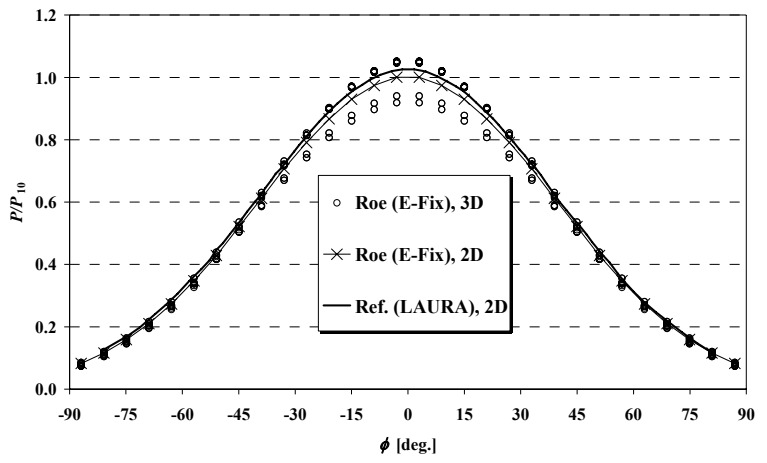

\section{b) Heating}

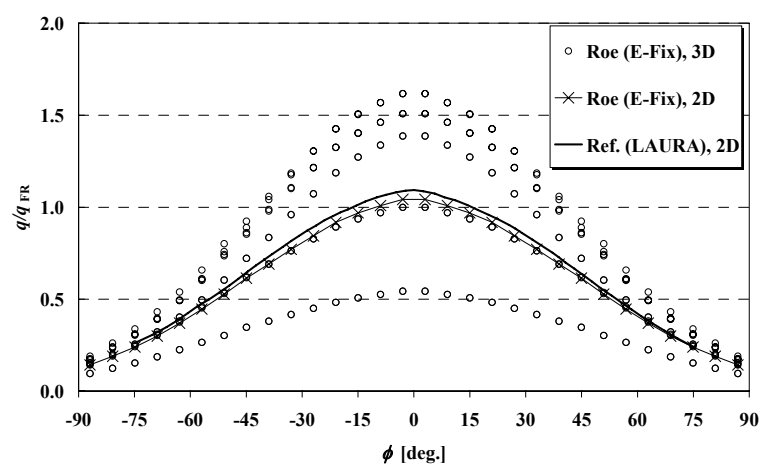

c) L2-norm of density residual

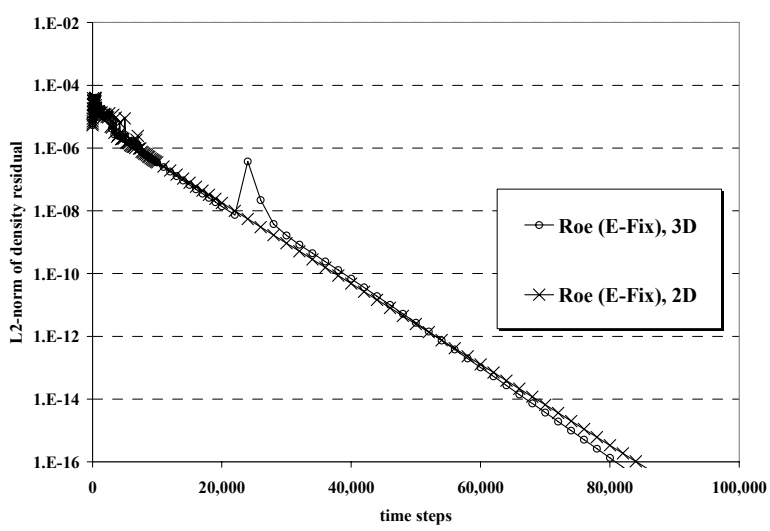

Figure 22. Surface pressure and heating profiles, and residual histories for cylinder (second-order in space; freestream Mach number $\left.M_{\infty}=12\right)$. [Roe (EFix), 3D grid] a) Pressure

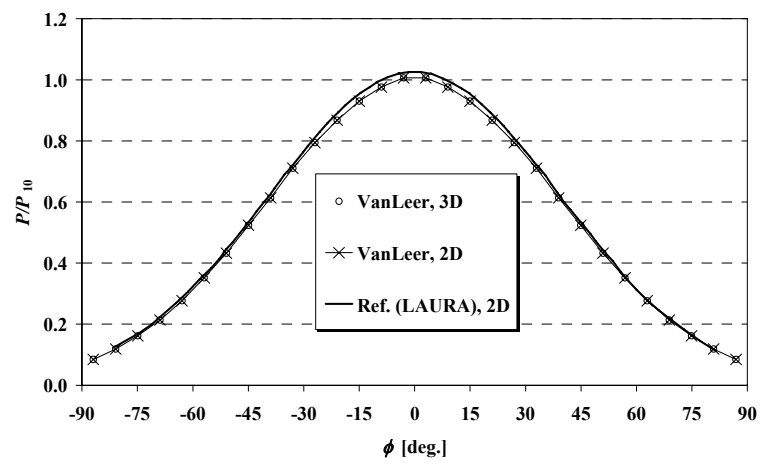

b) Heating

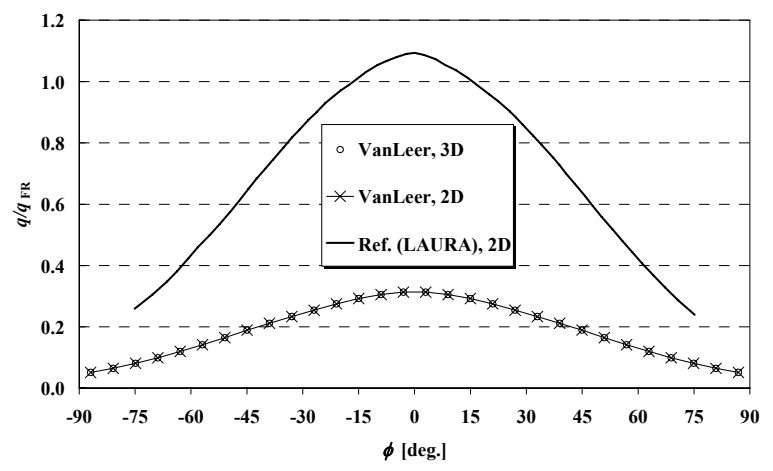

c) L2-norm of density residual

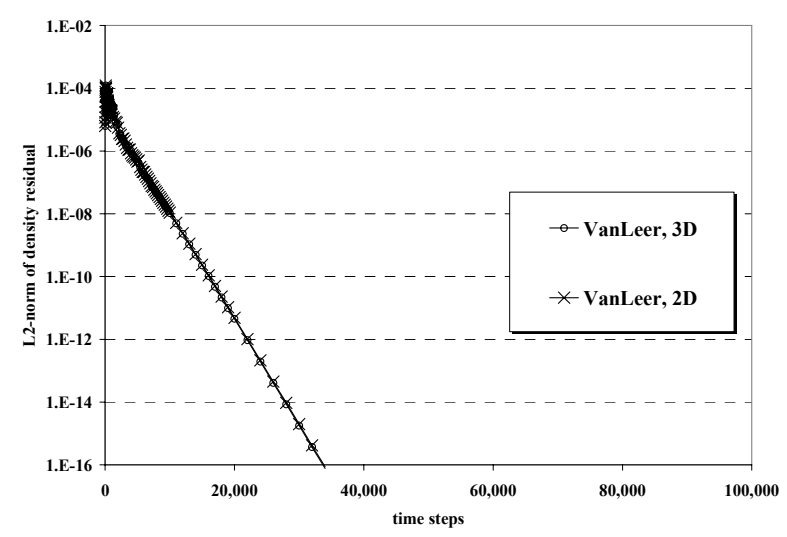

Figure 23. Surface pressure and heating profiles, and residual histories for cylinder (second-order in space; freestream Mach number $M_{\infty}=12$ ). [Van Leer, 3D grid] 
a) Pressure

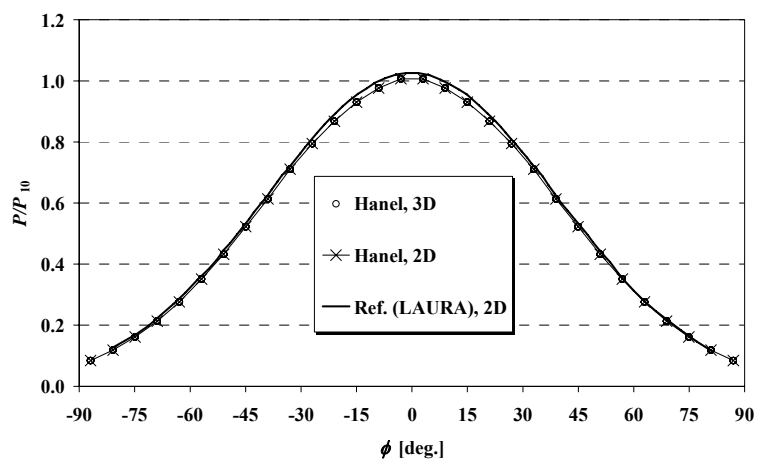

b) Heating

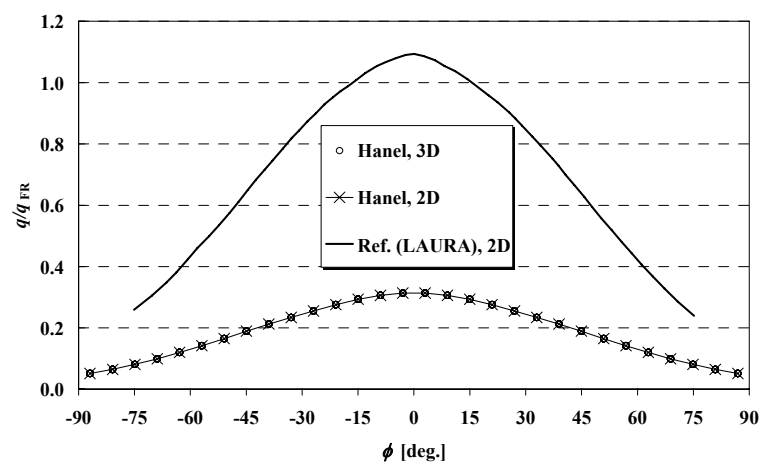

c) L2-norm of density residual

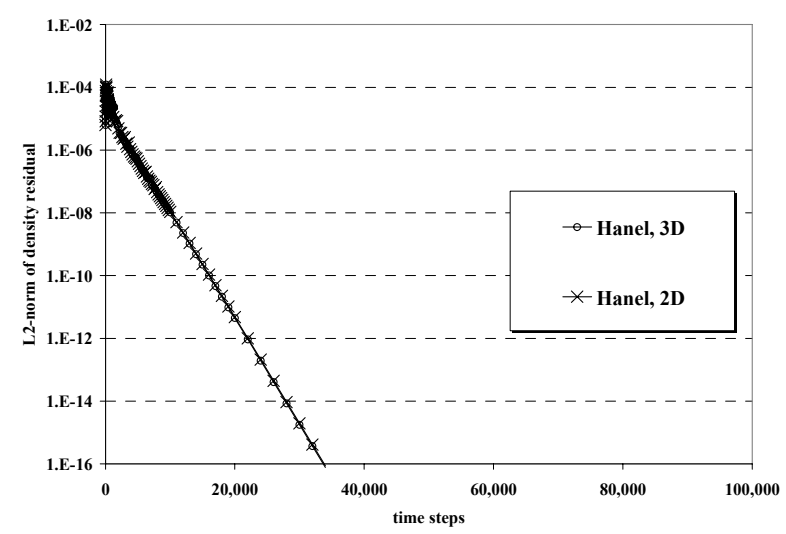

Figure 24. Surface pressure and heating profiles, and residual histories for cylinder (second-order in space; freestream Mach number $M_{\infty}=12$ ). [Hänel, 3D grid] a) Pressure

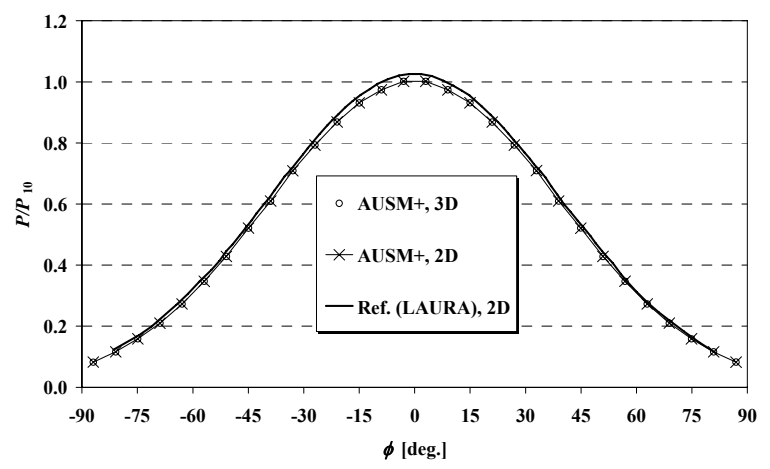

b) Heating

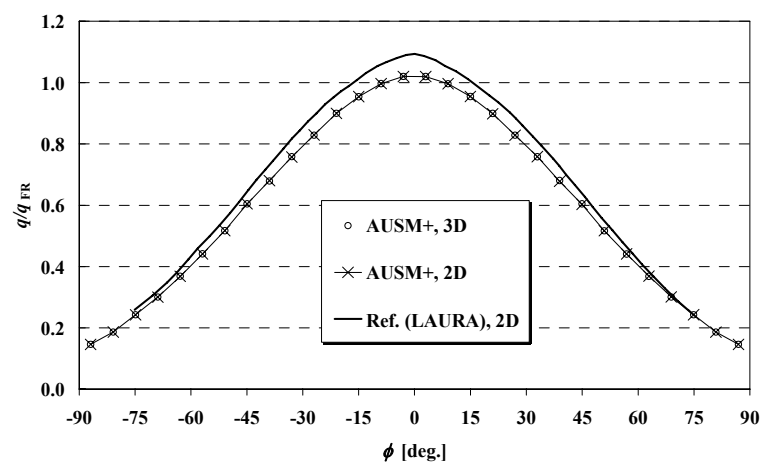

c) L2-norm of density residual

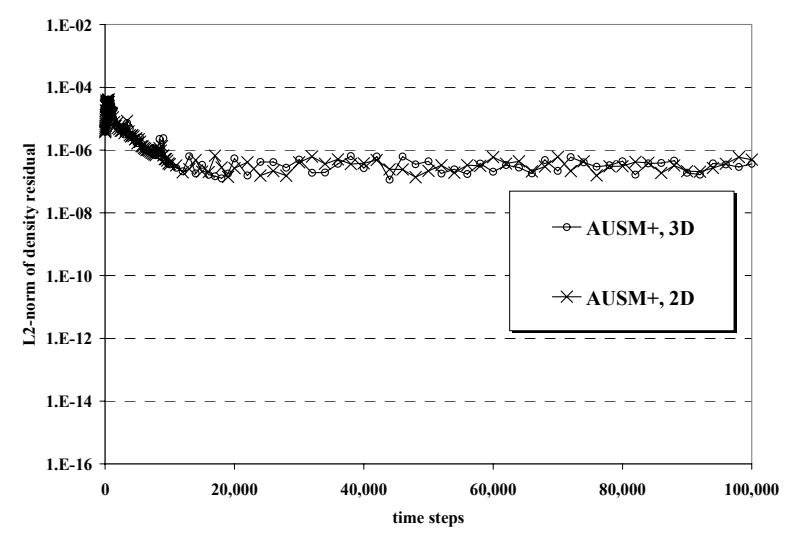

Figure 25. Surface pressure and heating profiles, and residual histories for cylinder (second-order in space; freestream Mach number $M_{\infty}=12$ ). [AUSM+, 3D grid] 
a) Pressure

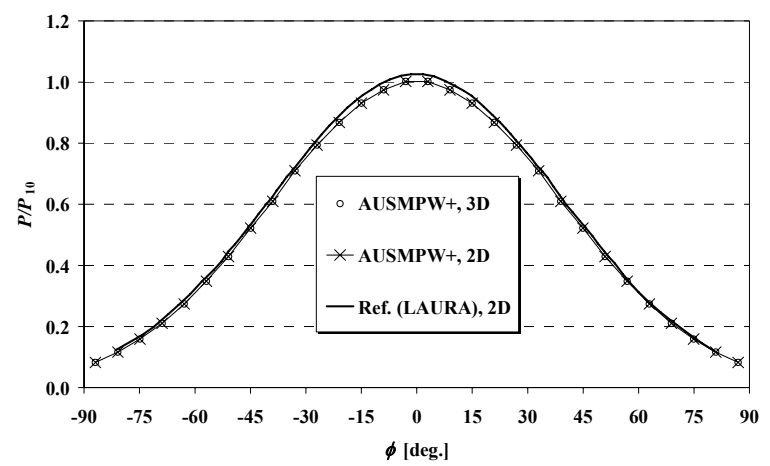

b) Heating

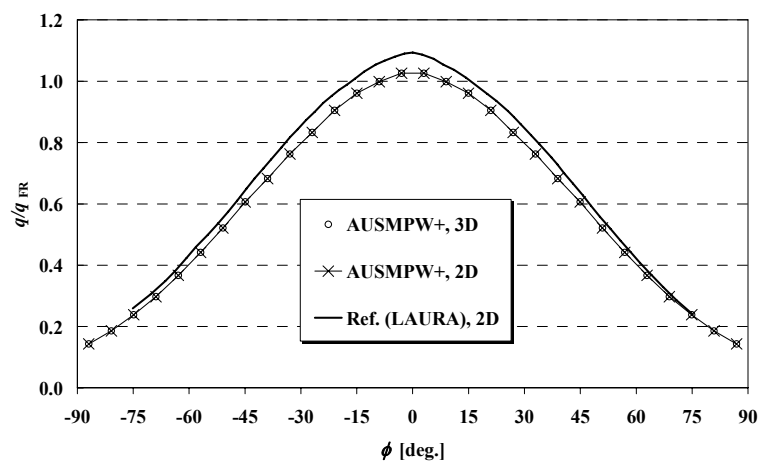

c) L2-norm of density residual

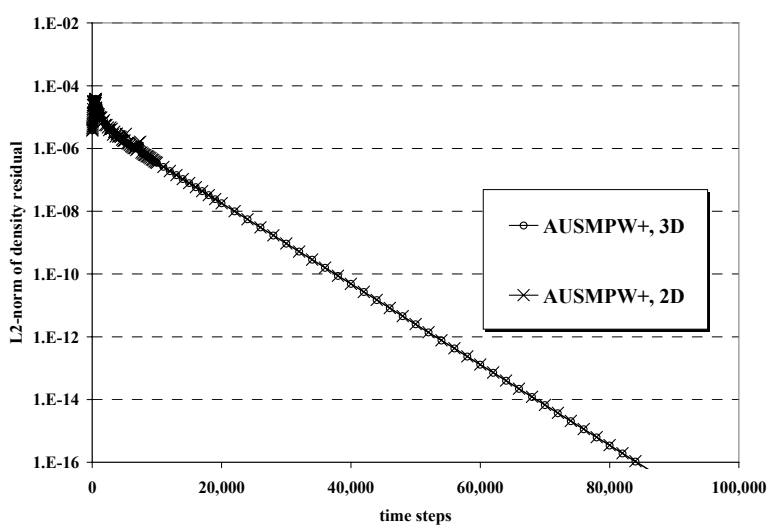

Figure 26. Surface pressure and heating profiles, and residual histories for cylinder (second-order in space; freestream Mach number $M_{\infty}=12$ ). [AUSMPW+, 3D grid] a) Grid

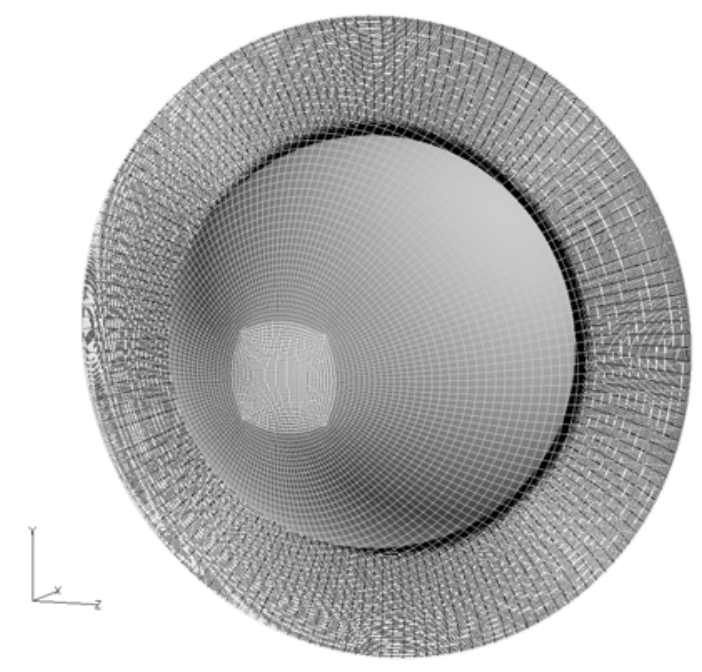

b) Pressure contours (AUSM+)

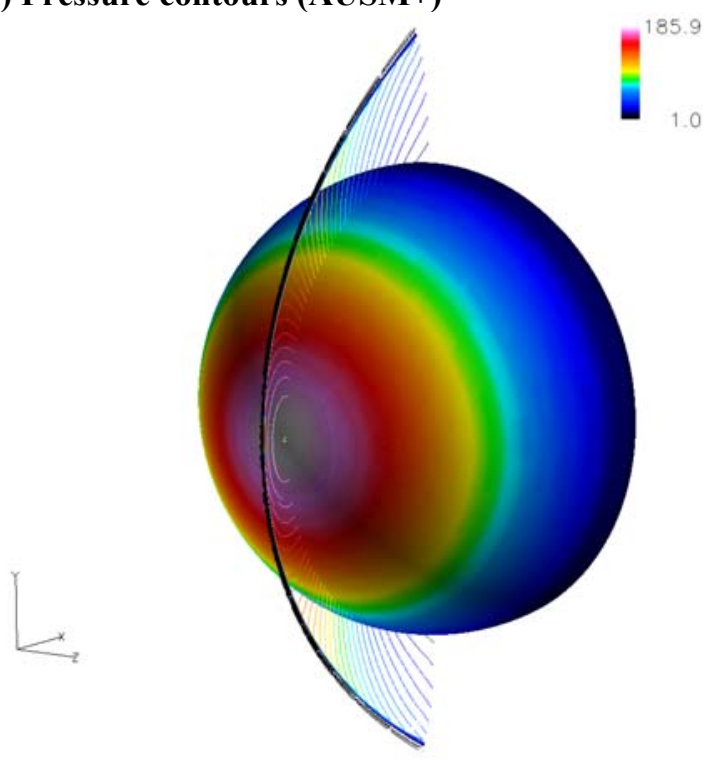

Figure 27. Computational grid and typical solution for $M_{\infty}=12$ Candler's 'challenge' problem. ${ }^{24}$ [3D grid] 
a) Van Leer
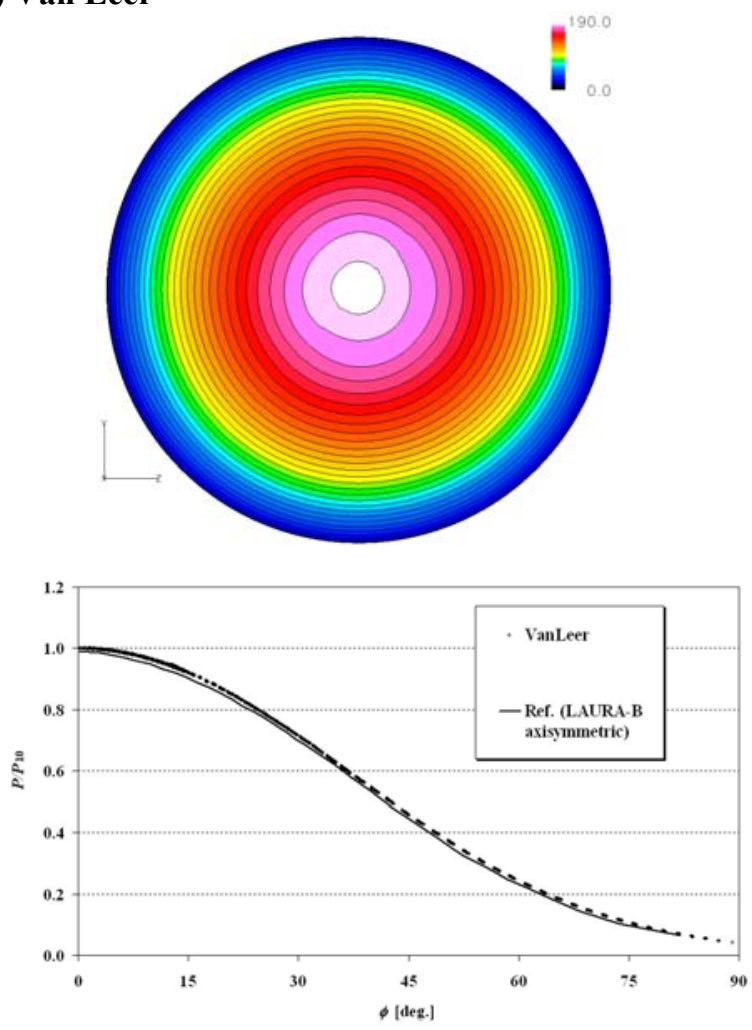

c) AUSMPW+
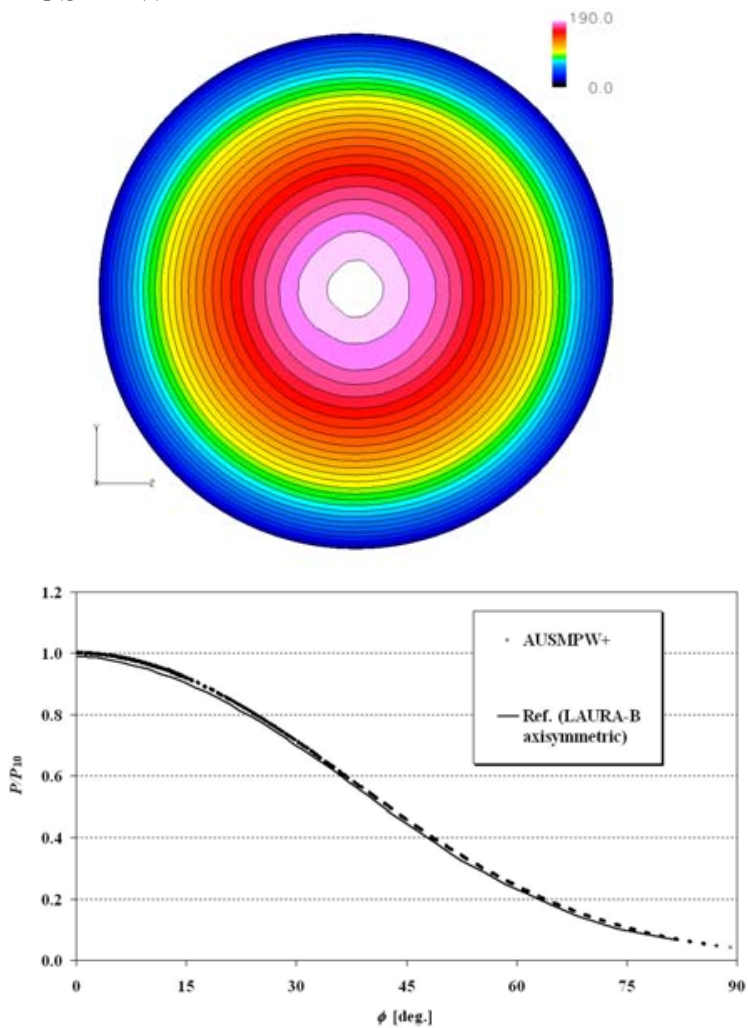

b) AUSM+
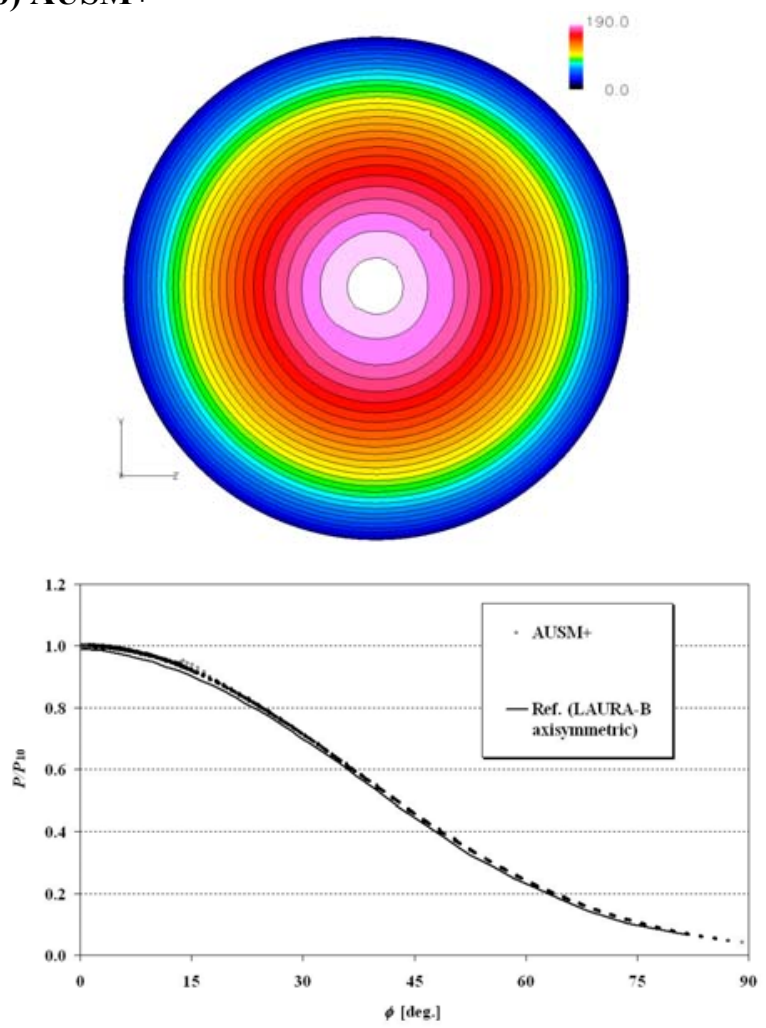

d) Ref. [9] Gnoffo
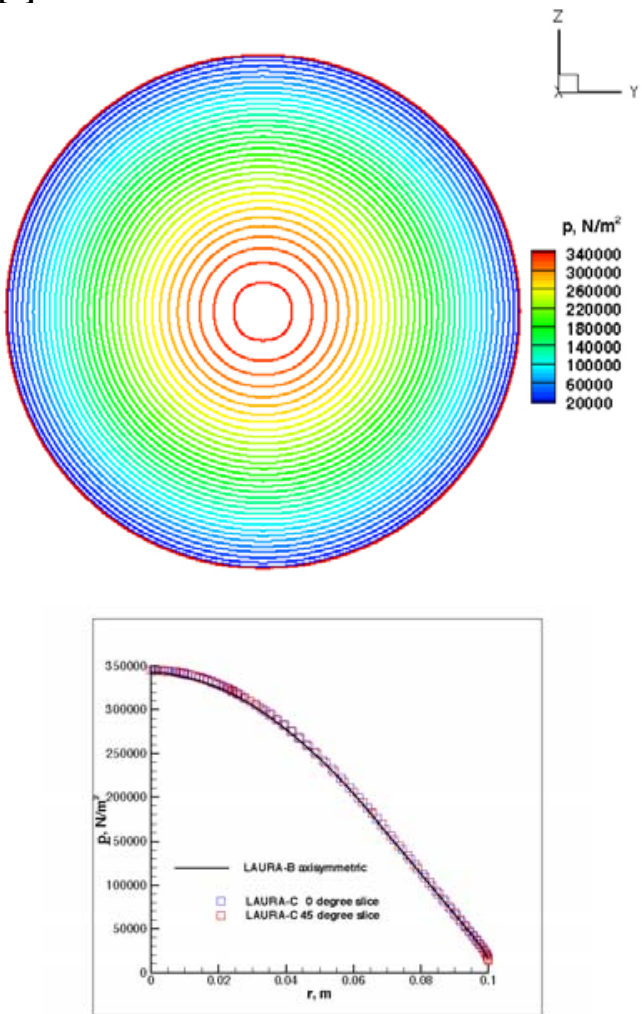

Figure 28. Surface pressure contours (top; $0<P / P_{\infty}<190$ ) and profiles (bottom) (3D, sphere). 
a) Van Leer
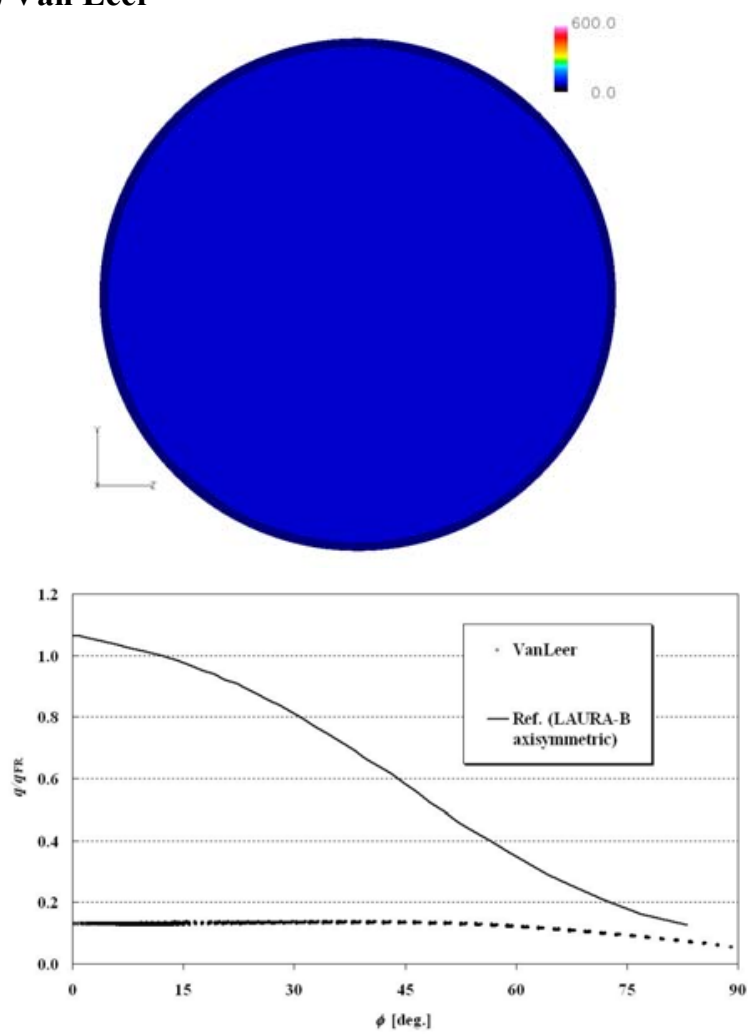

c) AUSMPW+
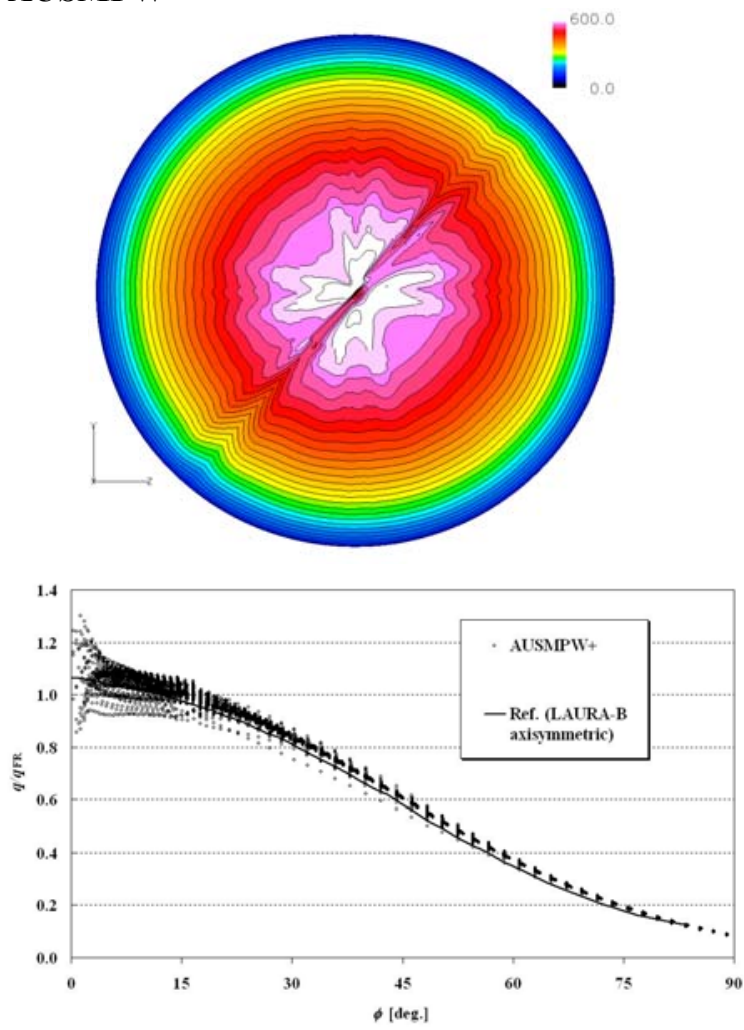

b) AUSM+
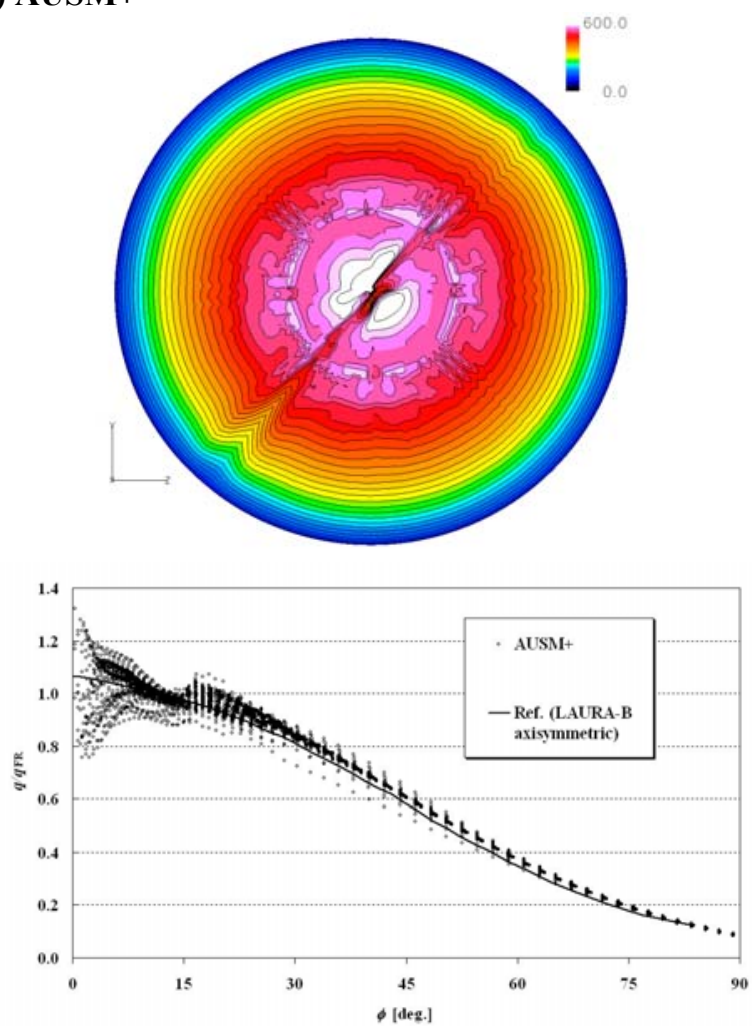

d) Ref. [9] Gnoffo
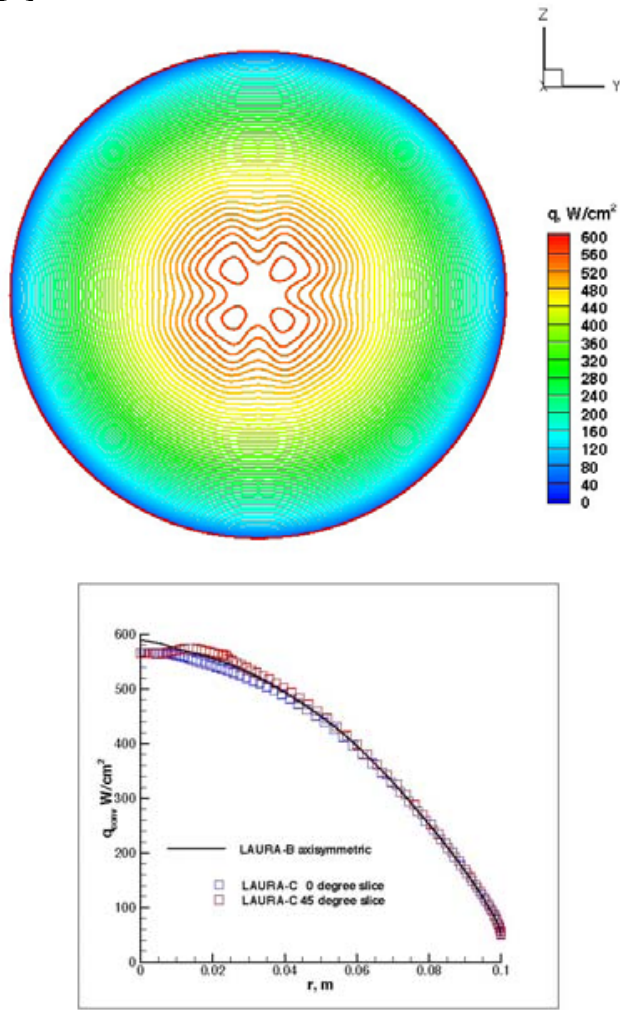

Figure 29. Surface heating contours (top; $0<q<600\left[\mathrm{~W} / \mathrm{cm}^{2}\right]$ ) and profiles (bottom) (3D, sphere). 


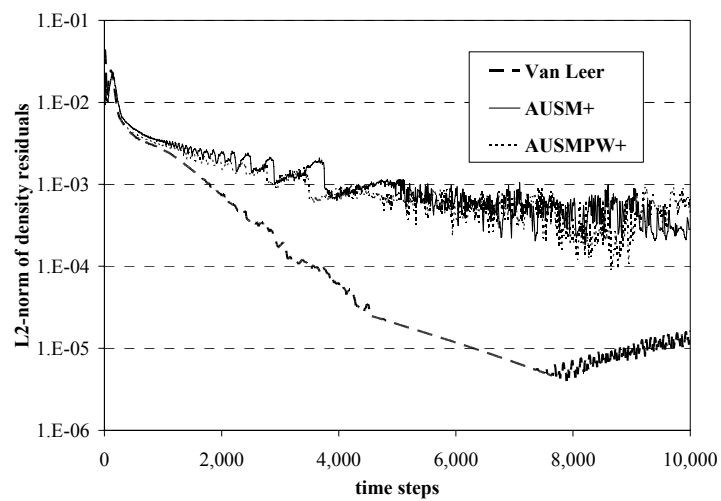

Figure 30. Residual histories for sphere $\left(M_{\infty}=12\right)$. [3D grid]

\section{Final Remarks}

We made a comparative study on the behaviors of flux functions with regard to two- and three-dimensional shock anomalies (instabilities and oscillations). The following features are noteworthy for hypersonic flow computations in three dimensions (3D):

1. A simple expectation that three-dimensional shock anomalies always appear more likely than twodimensional (2D) counterpart turned out to be false. Rather, the development of shock anomalies is seen in every direction and quite complicated in three dimensions. For instance, in a two-dimensional setup in three dimensions (circular-cylinder in 2D), computations reached the following solutions:

a. 3D case that exhibited carbuncle in 2D: The "carbuncle" developed in the two-dimensional slice, while the shock "oscillation" appeared in the third direction. These anomalies developed from the very begging of the computation with the same growth rate.

b. 3D cases that were stable in 2D: Depending on the cases of different grids or different flux functions, either the following two solutions were obtained: i) the totally symmetry solution, or ii) the stable, symmetry solution remained in the two-dimensional slice, whereas the shock "oscillation" developed in the third direction.

2. Multidimensional dissipations considered in AUSMPW+ flux function worked to suppress anomalous behaviors in limited cases, but not effective for genuinely two-dimensional or a genuinely three-dimensional development of shock anomalies.

3. AUSM-type fluxes generally yielded satisfactory predictions of heating for a two-dimensional problem in three dimensions for a cylinder, but not for a genuinely three-dimensional problem for a sphere.

It is demonstrated that multidimensional dissipation is effective, but not perfectly. This is partly because such dissipation terms had been developed under two-dimensional considerations, and partly because those terms do not always work successfully even in two dimensions. Consequently, a flux function showing good or fair robustness against the shock in two dimensions can either succeed or fail to reproduce acceptable solutions in three dimensions. Thus, when one attempts to test a flux function, it is recommended to keep it in mind that investigations only in two dimensions are not enough to accurately predict behaviors of the flux in three dimensions. In addition, although most of the existing Euler fluxes were designed based on one- or two-dimensions but readily extendable to threedimensions, it is desirable to invent a new method in consideration of the presented three-dimensional behaviors of the captured shock. If there is a flux function showing no anomalous solutions in all the tests presented here, the flux can be regarded as robust enough in three dimensions.

\section{Acknowledgments}

We are grateful to Peter Gnoffo, NASA Langley, for giving us computational grids used in the three-dimensional cases, and for permission to present his numerical results as reference. We also thank Jeffery White and Bil Kleb, NASA Langley, for providing us with grids. The computational code was originally developed at Nagoya University, Japan, while the first author was under supervision of Yoshiaki Nakamura. 


\section{References}

${ }^{1}$ Peery, K.M. and Imlay, S.T., "Blunt-Body Flow Simulations,” AIAA Paper 88-2904, 1988.

${ }^{2}$ Pandolfi, M. and D'Ambrosio, D., "Numerical Instabilities in Upwind Methods: Analysis and Cures for the "Carbuncle" Phenomenon," Journal of Computational Physics, Vol. 166, No. 2, 2001, pp.271-301.

${ }^{3}$ Kitamura, K., Roe, P., and Ismail, F., "Evaluation of Euler Fluxes for Hypersonic Flow Computations," AIAA Journal, Vol. 47, 2009, pp.44-53.

${ }^{4}$ Kim, K.H., Kim, C., and Rho, O.H., "Cures for the shock instability, Development of a shock-stable Roe scheme," Journal of Computational Physics, Vol. 186, 2003, pp.342-374.

${ }^{5}$ Kim, S.S., Kim, C., Rho, O.H., Hong, S.K., "Methods for the Accurate Computations of Hypersonic Flows I. AUSMPW+ scheme," Journal of Computational Physics, Vol. 174, 2001, pp.38-80.

${ }^{6}$ Nishikawa, H. and Kitamura, K., "Very Simple, Carbuncle-Free, Boundary-Layer Resolving, Rotated-Hybrid Riemann Solvers," Journal of Computational Physics, Vol. 227 (2008), pp. 2560-2581.

${ }^{7}$ Shima, E. and Kitamura, K. "On New Simple Low-Dissipation Scheme of AUSM-Family for All Speeds," AIAA Paper 2009-136, 2009.

${ }^{8}$ Roe, P.L. and Kitamura, K., “Artificial Surface Tension to Stabilize Captured Shockwaves,” AIAA Paper 2008-3991, 2008.

${ }^{9}$ Gnoffo, P.A., "Multidimensional, Inviscid Flux Reconstruction for Simulation of Hypersonic Heating on Tetrahedral Grids,” AIAA Paper 2009-599, 2009.

${ }^{10}$ Loh, C.Y. and Jorgenson, P.C.E., "Multi-dimensional Dissipation for Cure of Pathological Behaviors of Upwind Scheme," Journal of Computational Physics, Vol. 228 (2009), pp.1343-1346.

${ }^{11}$ Gnoffo, P.A. and White, J.A., "Computational Aerothermodynamic Simulation Issues on Unstructured Grids," AIAA Paper 2004-2371 (2004).

${ }^{12}$ Candler, G.V., Mavriplis, D.J., Treviño, L., "Current Status and Future Prospects for the Numerical Simulation of Hypersonic Flows,” AIAA Paper 2009-153, 2009.

${ }^{13}$ Kitamura, K., Shima, E., Nakamura, Y., and Roe, P., "Evaluation of Euler Fluxes for Hypersonic Heating Computations," AIAA Journal, (Accepted for Publication).

${ }^{14}$ Yoon, S.H., Kim, C., and Kim, K.H., "Multi-Dimensional Limiting Process for Three-Dimensional Flow Physics Analyses," Journal of Computational Physics, Vol. 227 (2008), pp.6001-6043.

${ }^{15} \mathrm{http}$ ://fun3d.larc.nasa.gov/chapter-9.html\#hypersonic benchmarks, retrieved on Jun. 4, 2009.

${ }^{16}$ Van Albada, G.D., Van Leer, B. and Roberts, Jr., W.W., "A Comparative Study of Computational Methods in Cosmic Gas Dynamics," Astron. Astrophys., Vol. 108 (1982), pp.76-84.

${ }^{17}$ Van Leer, B., "Towards the Ultimate Conservative Difference Scheme. V. A Second-Order Sequel to Godunov's Method," Journal of Computational Physics, Vol. 32 (1979), pp.101-136.

${ }^{18}$ Roe, P.L., “Approximate Riemann Solvers, Parameter Vectors, and Difference Schemes,” Journal of Computational Physics, Vol. 43 (1981), pp.357-372.

${ }^{19}$ A. Harten, "High Resolution Schemes for Hyperbolic Conservation Laws," Journal of Computational Physics, Vol. 49 (1983), pp.357-393.

${ }^{20}$ Van Leer, B., "Flux Vector Splitting for the Euler Equations," Lecture Notes in Phys., Vol. 170 (1982), pp. $507-512$.

${ }^{21}$ Hänel, D, Schwane, R., and Seider, G., "On the Accuracy of Upwind Schemes for the Solution of the Navier-Stokes Equations," AIAA Paper 87-1105, (1987).

${ }^{22}$ Liou, M.-S., “A Sequel to AUSM: AUSM+,” Journal of Computational Physics, Vol. 129 (1996), pp.364-382.

${ }^{23}$ Fay, J.A. and Riddell, F.R., "Theory of Stagnation Point Heat Transfer in Dissociated Air," J. Aeronautical Sciences, Vol. 25 (1958), pp.73-85.

${ }^{24}$ Candler, G., Barnhardt, M., Drayna, T., Nompelis, I., Peterson, D., and Subbareddy, P., "Unstructured Grid Approaches for Accurate Aeroheating Simulations," AIAA Paper 2007-3959, June 2007. 